\title{
The Two-Dimensional Spatial Structure of Simple Receptive Fields in Cat Striate Cortex
}

\author{
JUDSON P. JONES AND LARRY A. PALMER
}

Department of Anatomy, and David Mahoney Institute of Neurological Sciences, University of Pennsylvania School of Medicine, Philadelphia, Pennsylvania 19104-6058

SUMMARY AND CONCLUSIONS

1. A reverse correlation $(6,8,25,35)$ method is developed that allows quantitative determination of visual receptive-field structure in two spatial dimensions. This method is applied to simple cells in the cat striate cortex.

2. It is demonstrated that the reverse correlation method yields results with several desirable properties, including convergence and reproducibility independent of modest changes in stimulus parameters.

3. In contrast to results obtained with moving stimuli (26), we find that the bright and dark excitatory subregions in simple receptive fields do not overlap to any great extent. This difference in results may be attributed to confounding the independent variables space and time when using moving stimuli.

4. All simple receptive fields have subregions that vary smoothly in all directions in space. There are no sharp transitions either between excitatory subregions or between subregions and the area surrounding the receptive field.

5. Simple receptive fields vary both in the number of subregions observed, in the elongation of each subregion, and in the overall elongation of the field. In contrast with results obtained using moving stimuli (26), we find that subregions within a given receptive field need not be the same length.

6 . The hypothesis that simple receptive fields can be modeled as either even symmetric or odd symmetric about a central axis is evaluated. This hypothesis is found to be false in general. Most simple receptive fields are neither even symmetric nor odd symmetric.

7. The hypothesis that simple receptive fields can be modeled as the product of a width response profile and an orthogonal length response profile (Cartesian separability) is evaluated. This hypothesis is found to be true for only $\sim 50 \%$ of the cells in our sample.

\section{INTRODUCTION}

Vision is a multidimensional sensory modality. Even the simplified case of an achromatic image projected onto a single retina is a three-dimensional function of space and time. Accordingly, efforts to understand neural image processing have concentrated on the spatial and temporal structure of receptive fields at strategic points in the visual systems of cats and primates. These receptive fields generally cover only a small region of visual space; it is useful to think of them as local spatial operators, each simultaneously and continuously transforming local luminance distributions into trains of action potentials.

The orientation-selective simple cells first described by Hubel and Wiesel in their classic study of cat striate cortex (17) provide an outstanding example of such local spatial operators. These receptive fields are small and divided into elongated, parallel subregions alternately excited by stimuli brighter or darker than the field luminance.

However, quantitative studies of the spatial organization of simple receptive fields are typically one dimensional (1D). Numerous experiments employing broadside motion or stationary presentation of long bars 
and edges have demonstrated the periodic oscillation of excitability along the width axis of simple receptive fields $(3,9,21,26,27,29$, $30,34,40)$. Fewer quantitative 1D studies of the length axis of simple receptive fields have been undertaken $(15,32,41)$. Besides the work reported here, only the studies of Heggelund have been fully two dimensional (2D) (13).

The problem with $1 \mathrm{D}$ approaches to visual receptive-field structure is the set of a priori assumptions they make. Pairs of $1 \mathrm{D}$ experiments that establish length and width response profiles fully characterize simple receptive fields only if they are Cartesian separable. That is, the $2 \mathrm{D}$ response profiles $\mathrm{r}(x, y)$ must be of the form

$$
\mathrm{r}(x, y)=w(x) l(y)
$$

where $w(x)$ and $l(y)$ denote the response profiles obtained independently for the width and length axes of the receptive field. This assumption can only be tested with a full 2D experiment. Retinal images are spatially $2 \mathrm{D}$ and so are the monocular receptive fields of simple cells. Investigating the role simple cells play in spatial vision is inherently a $2 \mathrm{D}$ problem.

The purpose of the experiments described here is to generalize the quantitative mcasurement of simple receptive-field structure to two spatial dimensions. This goal led to the development of a new reverse correlation technique, which may be applied to any visual receptive field. With the use of this technique, we quantify that $2 \mathrm{D}$ spatial structure of simple receptive fields and test the conjecture that their receptive fields are Cartesian separable.

In the following paper (18), we generalize the quantitative measurement of simple rcceptive fields to 2D of spatial frequency and describe the 2D spectral organization of simple receptive fields. In the last paper of this series (18), we evaluate the hypothesis that simple receptive fields are linear filters with the same functional form as $2 \mathrm{D}$ Gabor filters $(5,24)$.

\section{METHODS}

\section{General}

Recordings were obtained from 14 adult cats. Each was initially anesthetized with an intraperitoneal injection of thiopental sodium $(40 \mathrm{mg} / \mathrm{kg})$.
A veinous cannula placed in each hindlimb permitted subsequent infusion of paralytic agents (tubocurarine chloride, $2 \mathrm{mg} / \mathrm{h}$; gallamine triethiodide, $15 \mathrm{mg} / \mathrm{h}$ ) and additional anesthetic (thiopental sodium, $2.5 \mathrm{mg} / \mathrm{h}$ ). A tracheal cannula was implanted, an initial bolus of $60 \mathrm{mg}$ gallamine was administered, and the animal was supported by positive-pressure respiration with end-tidal $\mathrm{CO}_{2}$ maintained at $4.0 \%$. Body temperature was held at $38^{\circ} \mathrm{C}$ by means of a self-regulating heating pad on which the animal lay. The head was supported by a bolt affixed to the skull over the frontal sinus. All stereotaxic attachments to the head were removed.

A modest craniotomy made over the right cerebral hemisphere at Horsley-Clark P2.0-4.0 was extended medially to expose the entire width of the saggital sinus. A small dissection of the dura was performed over the lateral gyrus. Electrodes entered the brain in a frontoparallel plane, but tilted $8^{\circ}$ from the vertical (so that the tip moved medially as the electrode advanced), to permit relatively long penetrations through area 17 . The craniotomy was covered with $4 \%$ agar-saline to minimize cardiopulmonary pulsations and to prevent drying.

Pupils were dilated with $1 \%$ atropine, nictitating membranes were retracted with phenylephrine hydrochloride, and plano contact lenses were inserted to protect the cornea. Streak retinoscopy was used to determine the refraction required to focus stimuli at $57 \mathrm{~cm}$ onto the retinas and spectacle lenses of the appropriate refractive power were placed in front of each eye. The refraction was verified physiologically, while recording from a simple cell early in the experiment, by measuring response modulation as a bivariate function of diopters of correction and spatial frequency of a drifting sinusoidal grating. The appropriate lenses were considered to be those that gave the highest spatial frequency cutoff for the cell. This procedure usually specified a range of lenses, and the midrange value was used. It was rarely necessary to change the lenses by more than 1 diopter from the values determined by retinoscopy.

Action potentials (spikes) were recorded with glass pipettes pulled to roughly $1-\mu \mathrm{m}$ tips, filled with $0.2 \mathrm{M} \mathrm{KCl}$, and beveled to 2-4 M . On occasion, tungsten-in-glass electrodes (23) were used. After several stages of amplification (band pass $100-3,000 \mathrm{~Hz}$ ) the signal was displayed on an oscilloscope and fed to a window discriminator. Bascd on multiple level and timing constraints the window discriminator signaled the occurrence of spikes to the computer (DEC PDP 11/40) with $50-\mu \mathrm{S}$ transitor transitor logic pulses, and to the oscilloscope with a brief beam intensification. The computer checked a double-buffered spike register during clock interrupt service routines, which occurred at least every millisecond in all routines. 
Following each experiment, a block was taken from the right occipital cortex, sectioned, and the sections were stained with cresyl violet. By using light microscopy, we confirmed that electrode penetrations were in area 17 . No attempt was made to mark recording sites or to determine their laminar positions.

\section{Stimulus presentation}

Stimuli were presented on a Tektronix 608 monitor (P-31 phosphor) supported in a motorized frame $57 \mathrm{~cm}$ from the cat. The monitor could be easily positioned anywhere within the central $25^{\circ}$ of the visual field. A mask was placed on the face of the monitor with a circular aperture $10 \mathrm{~cm}$ in diameter, giving an effective working area of $10^{\circ}$ of visual angle. The positions of the areae centralis were inferred from the projections of the optic discs determined by reversible ophthalmoscopy. Receptive-field locations were drawn on a clear plastic plate mounted in front of the monitor, which was removed during data acquisition. Receptive-field locations were traced onto sheets of paper at the end of each experiment.

Stimuli were generated using a modified Picasso cathode-ray tube (CRT) image generator (Innisfree) with all image parameters under computer control. Twelve-bit digital-to-analog converters (DACs) were used to control the stimulus $x$ and $y$ positions. Otherwise, all image parameters were controlled with eight-bit DACs. Some Innis- free functions were linear: $x$ and $y$ position, contrast, and orientation. These linear relationships were verified empirically. For other Innisfree functions, a nonlinear relationship existed between computer output and the resulting image parameter (spatial and temporal frequency, length, and width). The empirically measured nonlinear calibration curves were fitted with polynomials of up to the fifth order, using a leastsquared error algorithm. The functions obtained were stored in a subroutine library and used in all software for Innisfree control. Around the mean luminance $\left(22 \mathrm{~cd} / \mathrm{m}^{2}\right)$, the CRT was found to be linear within the range of $\pm 60 \%$ contrast $\left(\mathrm{L}_{\max }-\right.$ $\left.\mathrm{L}_{\min } / \mathrm{L}_{\max }+\mathrm{L}_{\min }\right)$, and this range was never exceeded.

\section{Procedures}

Once a single unit was isolated, a relatively fixed sequence of procedures was followed. By using a small hand-held projector, the dominant eye was determined and the opposite eye occluded with an opaque patch. The receptive-field location was marked on the plastic plate, the monitor was positioned behind the mark, the plate was removed, and the position of the monitor was refined, so that the entire receptive field was included on the monitor screen.

A computer-assisted handplotting program was used to determine a range of stimulus parameters for the subsequent quantitative analyses. This
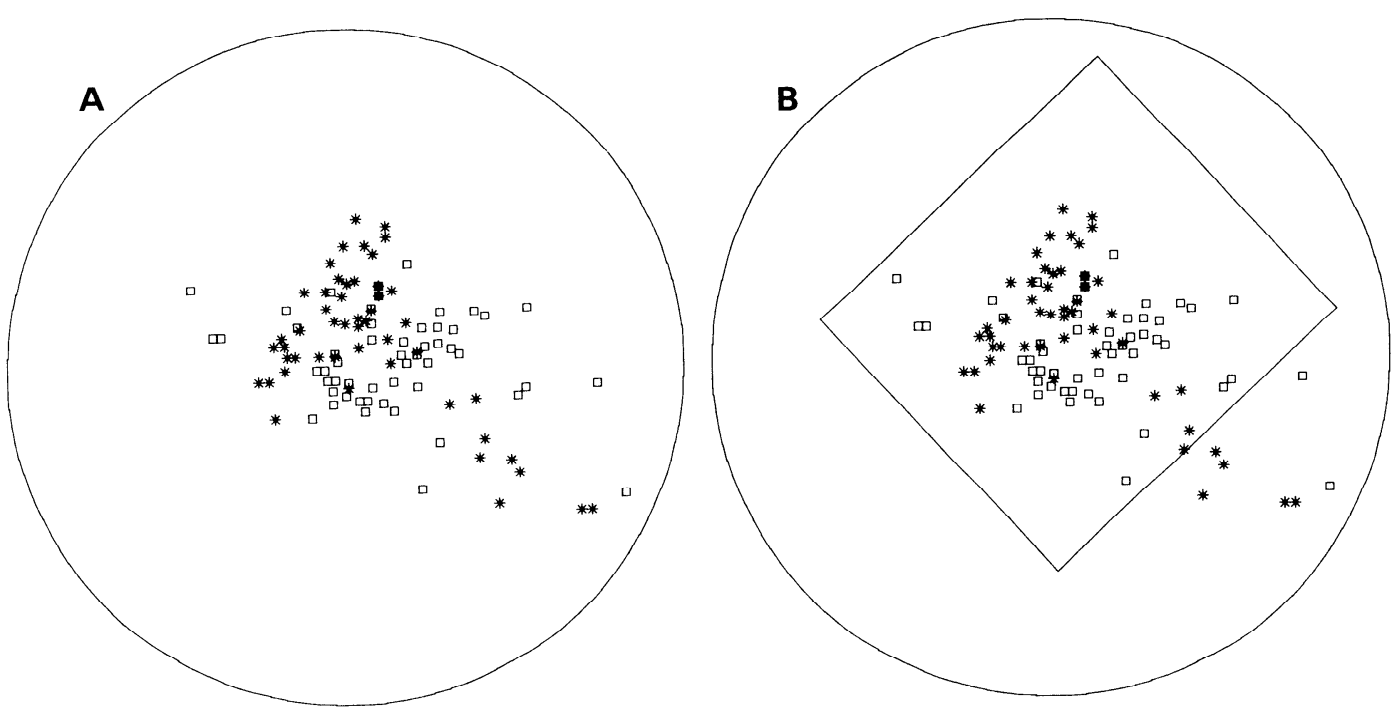

FIG. 1. A computer-assisted "handplotting" procedure. By means of hand-held potentiometers interfaced to the computer, the experimenter moved a bright or dark bar around the face of the oscilloscope, the limits of which are indicated by the large circle $\left(10^{\circ}\right.$ diam). When the neuron emitted a spike, a symbol was added to a computer graphic display at a location corresponding to the stimulus position on the oscilloscope. A: asterisks mark the positions of dark stimuli, and open squares mark bright stimuli. Dark stimuli eliciting spikes clustered in an elongated region to the upper left, and bright stimuli in an adjacent elongated region to the lower right. B: a rectangular box drawn around the receptive field was used for subsequent quantitative analysis. 
program was designed to quickly find and record the spatial locus or loci of evoked responses and to avoid subjective estimation of optimal stimulus parameters.

The handplotting program provided instantaneous graphical feedback of the stimulus positions that evoked spikes. The experimenter controlled stimulus length, width, contrast, orientation, position, and flicker frequency using potentiometers mounted on a hand-held box. The program relayed these parameters to the Innisfree, and displayed a line drawing of the stimulus, refreshed in real time, on a VT-11 vector graphic CRT screen. When a spike occurred, a symbol was added to the VT-11 screen at a position corresponding to the center coordinates of the current stimulus (Fig. $1 A$ ). Different symbols were used for spikes elicited by bright or dark stimuli. When recording from a simple cell, one could quickly determine the number, sign, and approximate spatial extent of individual subregions. The whole receptive field was then enclosed within a box drawn on the VT-11 screen, large enough to allow for possible responsive areas, too weak to be detected in the handplotting procedure, and oriented roughly parallel to the receptive-field modulation axis (Fig. 1B). The program saved the parameters of this box (location, length, width, and orientation) for use in the analysis described below.

Allowing the box variable orientation is equivalent to a rotation of the spatial coordinate axes and has the effect of roughly normalizing our results with respect to orientation. Variations in the orientation of the box away from the receptivefield elongation axis have no effect on subsequent analyses, since these routines did not assume a specific relationship between the orientation of the box and receptive-field structure. Receptive fields were classified as simple and accepted for subsequent analysis if this handplotting procedure indicated the existence of spatially segregated subregions with excitatory responses to stimulus contrasts of alternating sign, or if the cell produced a strongly modulated response to drifting sinusoidal gratings (see following paper and Ref. 14). These classification criteria correspond in the first case to one of Hubel and Wiesel's (13) defining characteristics, and in the second to that of Movshon et al. (27). No additional criteria were used (e.g., spatial summation within, or antagonism between, subregions). Provided that one of these criteria were satisfied, we also accepted as simple 1) cells exhibiting end stopping, although no effort was made to quantify the effect, and 2 ) cells with large receptive fields.

\section{Quantitative measurement of $2 D$ spatial receptive-ficld structure}

Typically, a neuron's response to a stimulus or set of stimuli is measured using one or more peri- stimulus time histograms (11). In this procedure, one presents a stimulus and then waits for the neuron to respond before presenting another stimulus. For the purposes of mapping the 1D spatial organization of simple receptive fields, the stimuli have traditionally been stationary flashing bars or moving bars or edges (see Orban for review, 31). We attempted to generalize the poststimulus time histogram method to two spatial dimensions using small bright and dark stationary flashing spots distributed on either a $16 \times 16$ grid or an $8 \times 8$ grid (a 2D generalization of the response plane technique of Stevens and Gerstein, 43), but we found that simple cells do not respond well enough to these stimuli to make this method practical.

The fundamental problem is that a stimulus of small energy (such as the small rectangle we need to make 2D measurements with moderate resolution) will, at best, cause only a small change in the probability of discharge. Therefore, we must average this change over many stimulus presentations to makc it obscrvablc. We nced many more stimuli than have been used in prior studies, and to make the method practical, we must present these stimuli in $1 \mathrm{~h}$ or less. Because the stimuli must therefore be presented rapidly, we must also control for the interaction between nearby stimuli.

The key features of the method we now present are rapid presentation of many small, uniformly distributed, bright and dark stimuli, and crosscorrelation between this random stimulus ensemble and the spike train. Because the stimuli are very small, we get the spatial resolution we need. Because the stimuli are presented rapidly, we are able to deliver enough of them to observe the small change in the probability of discharge they produce. Because the stimuli are randomly distributed (2D uniform distribution), there is no average interaction between stimuli. We crosscorrelate the 2D spatial distribution of stimuli with the spike train after a fixed delay to recover the probability that a given change of contrast over a given small area will cause a spike.

In these experiments, the following parameters were fixed: the stimuli were always distributed on a $16 \times 16$ rectangular grid of the same size and orientation as the box determined in the handplotting routine, and they always had a $60 \%$ contrast. This gave 512 distinct stimuli: $16 \times 16$ or 256 bright stimuli, and $16 \times 16$ or 256 dark stimuli. Stimuli were always exactly centered on the points in the sampling grid. Stimuli were always rectangular, and their sizes were determined in terms of grid units where a length grid unit was $1 / 16$ of the full length of the grid and a width grid unit was $1 / 16$ of the full width. Stimuli were presented one at a time, and their duration was always some integral multiple of $5 \mathrm{~ms}$, usually 50 or $100 \mathrm{~ms}$. Cross-correlation was done only over time delays 
equal to integral multiples of the stimulus duration. Nonzero time lags will be considered later to account for response latencics, but to kecp the description of the method simple, we will initially consider only one case of reverse correlation for zero latency.

Each of the stimuli was represented with a nine-bit code: four bits for the $x$ location in the grid, four bits for the $y$ location, and one bit for the contrast (bright or dark). These codes were assembled in random order in a long list $(5,632$ entries), which was guaranteed to contain an equal number of instances of each stimulus. The stimuli appeared in a different order each time the program ran. Each entry in the stimulus list was identified by a unique integer index (or address). The indices of the stimuli were in strictly increasing order from 1 to 5,632 .

Successive stimulus codes were drawn from the list at a constant rate (typically every $50 \mathrm{~ms}$ ), decoded, and sent to the image generator. Every 50 ms a new stimulus appeared on the CRT screen. (Viewing this is like viewing a light snowfall, provided that one permits black flakes as well as white ones.) When the program reached the end of the stimulus list, it started over again at the beginning (circular buffering). The clock interrupt service routine executed every $500 \mu \mathrm{s}$, checking for "time to present new stimulus", and checking for spikes.

When a spike occurred, the program saved the index of the stimulus then being displayed (a number between 1 and 5,632) in a list we call the spike list. The appropriate way to think about this list is that it contained the indices of (or the addresses of) the set of stimuli which were present when spikes occurred. The spike list was large enough to hold 8,192 entries.

When data acquisition was completed (when the spike list was full, or on a signal from the experimenter) there were two long lists in the computer's memory. The stimulus list encoded the spatial locations and contrasts of 5,632 stimuli; the spike list encoded which stimuli were present when the cell fired spikes.

Given these two lists it was possible to determine the set of stimuli that were present when the neuron emitted spikes. A subroutine was invoked that examined each entry in the spike list, found the corresponding stimulus code, and resolved it into its $x, y$, and contrast components. Then the subroutine added one to a 2D array (a grid) at an indexed position corresponding to the $x, y$ position of the stimulus. Two 2D grids were maintained: one for bright stimuli, and one for dark stimuli. Following the nomenclature established by DeBoer (6), we refer to this process as "reverse correlation", and the subroutine that implements it as the "reverse correlator."

Figure 2 illustrates the procedure; time moves left to right. The stimulus list contains descriptions of successive stimuli in terms of their contrasts (bright or dark), and $x$ and $y$ positions (numbers from 1 to 16 denote positions within the grid; the length, width, orientation, and duration of all stimuli are the same, and established in advance). Successive stimuli are identified by successive addresses. Assuming that the stimulus rate has been established at $50 \mathrm{~ms}$ per stimulus $(20$ $\mathrm{Hz}$ ), every $50 \mathrm{~ms}$ the clock interrupt service routine retrieves a stimulus code and delivers the corresponding stimulus parameters to the image generator. When a spike occurs (spike train in Fig. 2 ), the clock interrupt service routine records the address of the stimulus currently being delivered in the spike address list.

The reverse correlator starts at the beginning of the spike list, and for every entry it finds there, it retrieves the corresponding stimulus code. Based on the stimulus contrast, it selects the appropriate grid (bright or dark) and increments a grid location corresponding to the stimulus $x$ and $y$ position. Several examples of this decode and increment operation are shown in Fig. 2, including an example of two spikes that occurred during the same stimulus. When this process is complete (after several thousand spikes) the bright and dark grids contain counts of the stimuli that were on the oscilloscope screen at the same time spikes occurred.

Given this process, it was possible to determine not only the set of stimuli that were present when the cell spiked, but also the set of stimuli that were present prior to the occurrence of spikes in integral multiples of the stimulus duration. For each entry in the spike list, the reverse correlator found the stimulus that was present one stimulus interval before the spike by subtracting one from the stimulus index for that spike. This stimulus was decoded, as above, and processed into the bright and dark stimulus grids. After cvery spike in the spike list was processed, the two grids contained the spatial distribution of stimuli that preceded spikes by one stimulus interval; one grid contained the bright stimuli, the other contained the dark stimuli.

One further enhancement was necessary to make this method practical. Early in the experimental series, we often found that the stimuli did not evoke spikes quickly enough. Therefore, we made the stimuli larger by a selectable odd-integral multiple of the grid unit size. The reverse correlation proceeded as before, except that each time a stimulus was associated with a spike, the reverse correlator added one to the coordinate specified by the stimulus descriptor, and also added one to the surrounding bins corresponding to the stimulus size within the grid. A modest increment in the stimulus size, to $1 \times 3$ grid units as opposed to $1 \times 1$ grid units, was sufficient to give 


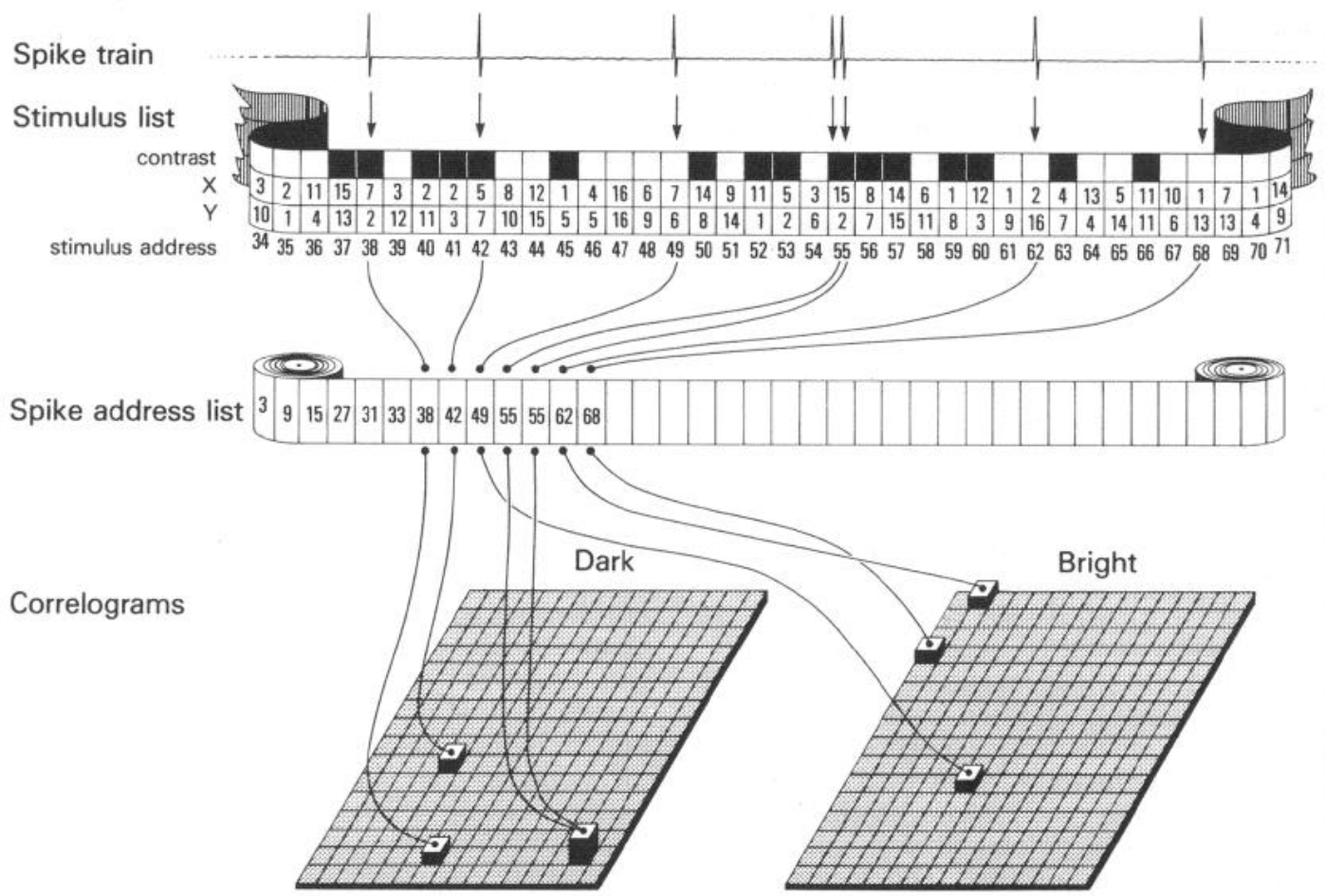

FIG. 2. Diagram of the reverse correlation procedure. Stimulus contrasts and positions are encoded in a long stimulus list, where the stimulus parameters occur in random sequence. Each stimulus has a unique address. When a spike occurs (spike train), the clock interrupt service routine records the stimulus address in a separate list, the spike address list. The reverse correlator decodes the entries in this list and increments locations in 2 separate grids based on the contrast and spatial position of each stimulus.

more rapid data acquisition. Most of the data reported in this paper were collected with stimuli that were one width grid unit and three length grid units in size (in a few cases, $1 \times 1$ and $1 \times 5$ stimuli were used).

The cost of this enhancement was added complexity in the reverse correlator routine, and a slight complication in the interpretation of the data on the edges of the grid. That is, given a $1 \times 3$ stimulus, when each stimulus was presented once on the grid, the stimulus fell on each grid point three times, except for the outermost row of points in the three-wide direction. These points were covered only twice (once when the stimulus center coordinates were in that row, and once when the stimulus center coordinates were adjacent to that row). In response to this situation we had two choices: we could multiply each outer row in the grid by $3 / 2$ to normalize for this effect, or we could do nothing, while keeping in mind that the outermost rows of the grid contain data that require additional interpretation. Because many of the receptive fields we examined did not en- croach on these outer rows, we adopted the second approach.

Deriving the 2D spatial response profile from the above data is straightforward. Provided that the response to contrast is linear about the field luminance in the range we are studying (near threshold), we can subtract the response to dark stimuli from the response to bright stimuli to estimate the inhibitory response to bright stimuli (27). Because simple cells typically have little or no spontaneous activity, we are required to invoke this operation. There is an added benefit: after subtracting the dark stimulus response from the bright stimulus response, the resulting difference grid contains unique values for each point; it is a function of two spatial variables. It is this difference that we call the "2D spatial response profile" of the receptive field.

Although we have presented stimulus presentation, data acquisition, and reverse correlation as separate processes, in practice they all occur simultaneously. During an experiment the program presents stimuli, acquires spikes, and computes 
and displays the accumulating reverse correlation at any desired stimulus interval in real time. In addition, the experimenter can select display of the correlation with bright stimuli only, the correlation with dark stimuli only, or the difference between these two correlations. Because the cell does not respond with large high-frequency bursts during this experiment (it sounds instead like spontaneous activity), instantaneous graphical feedback is a very useful diagnostic.

The method we have described here is but an approximation to the ideal case in several respects. Ideally, one would have an infinitely long list of stimulus codes, so that one would never repeat a sequence. This cannot be achieved on a finite-state machine like a digital computer. Ideally, one would like the experiment to run forever, so that one could guarantee that the measurement converged to the true receptive-field structure. This cannot be achieved for several self-evident reasons. Ideally, one would like to use spatially infinitesimal stimuli presented for an infinitesimal time on a continuum, rather than on a grid.

We can make a few statements concerning our particular choices for the design of this method. First, it can be shown that the effect of finite (as opposed to infinitesimal), rectangular stimuli has the effect of converting the three-dimensional space-space-time power spectrum of the stimulus ensemble from flat (whitc) to "sinc squared", with the half-power point in frequency inversely proportional to the stimulus size in space and time. Thus it is to our advantage to choose small, brief stimuli to move this attenuation point beyond the neuron's cutoff region. Second, the effect of repeating the stimulus sequence is to chop the stimulus spectrum into a series of finely spaced spectral lines whose spacing in frequency is inversely proportional to the period of repetition. Note that a list of 5,632 50-ms stimuli repeats itself only once every $4 \mathrm{~min} 41.6 \mathrm{~s}$. Third, the effect of using one stimulus at a time (as opposed to many) is simply to reduce the overall power in the ensemble.

Developing this method required many design decisions. In optimizing any one part of the design, we had to avoid making any other part absurd. We believe we have developed a useful and practical method for measuring the $2 \mathrm{D}$ spatial response profiles of simple cells, despite its flaws. Presumably, this method could be applied to other visual neurons. A more rigorous discussion of this method and empirical evaluation by simulation are given elsewhere (35).

\section{Nomenclature and conventions}

The method described in this paper yields a set of measurements we call the $2 \mathrm{D}$ spatial response profile. We chose the word profile because we wished to explicitly recognize that receptive fields are multidimensional (at least 2D of space plus time), and the term profile reflects the fact that our measurements represent $2 \mathrm{D}$ sections through a higher dimensional entity.

We refer to individual subregions in simple receptive fields as either "bright excitatory" or "dark excitatory" to denote a positive correlation between spikes and either bright or dark stimuli in a particular region of space. We avoid the terms excitatory and inhibitory because we cannot quantify inhibition in extracellular recordings. We avoid ON and OFF because the stimuli are bright and dark and our measurements are not taken relative to stimulus transitions.

None of the data in this paper have been subjected to filtering, smoothing, or any other kind of enhancement operation for the purposes of either analysis or presentation. We note, however, that there is some inherent signal averaging in our implementation of the reverse correlation procedure in the sense that adjacent stimuli overlap in the length direction (by $2 / 3$ for the most common case of a $1 \times 3$ stimulus). This has the same effect as a boxcar filter on the output; the signal is a bit smoother and a bit spread out in the length direction. In the width direction, all of the samples are independent. There is some further signal averaging in the sense that each stimulus was presented many times. The decision to stop data collection was made qualitatively, that is, when it was observed that the results were not "changing much."

The isoamplitude contour plots presented in this and the accompanying papers were drawn using the algorithm of Snyder (42). Our implementation of this algorithm uses linear interpolation between points on the grid, hence the piecewise linear appearance of the plots. In the contour diagrams, loci of equal response amplitude are connected so that the full response range (maximum to minimum response amplitude) is divided into either 6 intervals (all positive data) or 12 intervals (signed data). The contours are drawn around normalized surfaces at $\pm 10,30,50,70$, and $90 \%$ of the extreme absolute value. Contours above zero are drawn with solid lines, and contours below zero are drawn with short dashed lines. The 50\% contours both above and below zero are drawn with long dashed lines. These choices are arbitrary, but yield graphs that are informative without being too cluttered. The grid surfaces presented in these papers were prepared with the aid of Anderson's algorithm (2). The 2D spatial response profiles of 20 of the 36 cells we examined are illustrated in various contexts in this paper and the third paper of this series. As an aid to cross-referencing these data, 2D spatial response profiles are identified by name and associted with figure numbers and stimulus parame- 
TABLE 1. Stimulus parameters and figure cross-references for illustrated $2 D$ spatial response profiles

\begin{tabular}{|c|c|c|c|c|c|c|c|}
\hline \multirow[b]{3}{*}{ Cell } & \multirow[b]{3}{*}{$\begin{array}{l}\text { No. of } \\
\text { Spikes }\end{array}$} & \multicolumn{3}{|c|}{ Stimuli } & \multicolumn{2}{|c|}{ Grid } & \multirow[b]{3}{*}{ Figs. } \\
\hline & & \multirow[b]{2}{*}{ No. } & \multirow[b]{2}{*}{$\begin{array}{c}\text { Time } \\
\text { ms }\end{array}$} & Size, deg & Size, deg & \multirow[b]{2}{*}{$\begin{array}{c}\text { Orientation, } \\
\text { deg }\end{array}$} & \\
\hline & & & & $\begin{array}{ll}\mathrm{w} & 1\end{array}$ & w & & \\
\hline 0805 & 213 & 5,395 & 75 & $1.7 / 2.8$ & $9.0 / 6.6$ & 82 & $7 C$ \\
\hline 0107 & 1,221 & 21,794 & 100 & $0.4 / 2.1$ & $6.7 / 6.7$ & 64 & $7 B$ \\
\hline $0511-B$ & 8,192 & 24,909 & 50 & $0.4 / 1.1$ & $5.5 / 5.5$ & 127 & $5 E$, top $(2 E, 3 E)$ \\
\hline $0511-C$ & 4,937 & 5,633 & 100 & $0.4 / 1.1$ & $5.5 / 5.5$ & 127 & $5 E$, middle \\
\hline $0511-D$ & 5,621 & 11,266 & 100 & $0.4 / 1.6$ & $5.5 / 8.1$ & 127 & $5 E$, bottom, 10 \\
\hline 0711 & 453 & 11,720 & 50 & $0.2 / 0.6$ & $3.2 / 3.3$ & 146 & $6 C$ \\
\hline $0811-A$ & 226 & 5,633 & 50 & $0.2 / 1.2$ & $3.8 / 6.1$ & 98 & $5 B$, top \\
\hline $0811-B$ & 657 & 16,934 & 50 & $0.1 / 1.2$ & $2.0 / 6.1$ & 98 & $5 B$, bottom \\
\hline $0612-A$ & 3,649 & 11,266 & 50 & $0.3 / 1.1$ & $5.0 / 5.5$ & 147 & $5 C$, top \\
\hline $0612-B$ & 5,716 & 11,266 & 50 & $0.4 / 1.3$ & $6.0 / 6.7$ & 127 & $5 C$, bottom \\
\hline 0314 & 2,632 & 22,532 & 50 & $0.4 / 0.9$ & $5.9 / 4.6$ & 178 & $8 D(4 B)$ \\
\hline $0414-A$ & 1,640 & 11,265 & 50 & $0.4 / 1.2$ & $7.1 / 6.1$ & 33 & $5 D$, top \\
\hline $0414-B$ & 3,873 & 50,697 & 50 & $0.4 / 0.5$ & $7.1 / 7.1$ & 18 & $5 D$, bottom \\
\hline 0415 & 1,175 & 28,697 & 50 & $0.3 / 0.9$ & $4.8 / 4.8$ & 108 & $12 C$ \\
\hline 0116 & 8,192 & 26,423 & 50 & $0.4 / 1.7$ & $8.7 / 8.7$ & 258 & 4 \\
\hline 0316 & 8,192 & 29,971 & 100 & $0.3 / 1.8$ & $5.0 / 8.9$ & 102 & $8 A, 12 B(2 A, 3 A)$ \\
\hline 1316 & 444 & 32,980 & 50 & $1.0 / 2.3$ & $5.3 / 7.5$ & 78 & $7 A$ \\
\hline 0218 & 8,192 & 30,366 & 100 & $0.4 / 1.6$ & $5.5 / 7.9$ & 111 & $8 B(2 B, 3 B, 14)$ \\
\hline 0219 & 8,192 & 41,145 & 50 & $0.2 / 0.6$ & $3.9 / 3.0$ & 52 & $7 D, 12 D(2 D, 3 D)$ \\
\hline 0319 & 8,192 & 18,299 & 50 & $0.6 / 1.4$ & $9.4 / 7.1$ & 174 & 3 \\
\hline 0619 & 1,377 & 25,477 & 50 & $0.6 / 1.6$ & $8.9 / 8.0$ & 1 & $6 B(4 C)$ \\
\hline 0719 & 2,669 & 38,281 & 50 & $0.4 / 1.6$ & $6.1 / 8.0$ & 122 & $1,12 A$ \\
\hline 0221 & 513 & 11,827 & 100 & $0.9 / 2.4$ & $4.4 / 7.1$ & 46 & $8 C(2 C, 3 C)$ \\
\hline $0122-A$ & 1,887 & 24,843 & 50 & $0.6 / 2.0$ & $9.6 / 9.9$ & 46 & $5 A$, left \\
\hline $0122-B$ & 7,145 & 60,148 & 50 & $0.6 / 2.0$ & $9.6 / 9.9$ & 39 & $5 A$, right, $11(2 F, 3 F)$ \\
\hline 0224 & 4,888 & 45,046 & 50 & $0.6 / 1.5$ & $9.5 / 7.2$ & 97 & $6 A(4 A)$ \\
\hline
\end{tabular}

Profiles are identified by an arbitrary numerical designation; multiple profiles taken from single cells are given alphabetic extensions. Figure numbers in the 3rd paper of this series (18) are in parentheses. Stimulus parameters are given in terms of the number of stimuli delivered (no.), the temporal duration of each stimulus, (time), and the width (w) and length (l) of individual stimuli in degrees of visual angle. These stimuli were distributed over a two-dimensional (2D) spatial grid, whose parameters are given in terms of width and length in degrees of visual angle, and orientation (of the width axis) in degrees counterclockwise from horizontal. The number of spikes contributing to the illustrated profiles is also indicated (no. of spikes).

ters in Table 1. Stimulus parameters are not repeated in the individual figure legends.

\section{RESULTS}

This report is based on $552 \mathrm{D}$ spatial response profiles obtained from 36 simple cells in 14 cats.

\section{Part I. methodological considerations}

GROWTH AND CONVERGENCE OF THE REVERSE CORRELATION. Because the stimulus list and the spike list were preserved following each experiment, it was possible to reconstruct an experiment spike for spike and study the reverse correlation as it evolved. As an example, we consider this evolution in some detail for a single case, illustrated in Fig. 3.

The corrclations with bright stimuli, dark stimuli, and their difference are shown at a very early stage of the experiment in Fig. $3 \mathrm{~A}$, after only 64 spikes had occurred. No receptive-field structure is yet evident. An example of the effect of a single spike is marked with an arrow in the correlation with dark stimuli. The spike produces a small deflection in the surface in three adjacent locations, reflecting the fact that the stimulus was $1 \times 3$ grid units in size. In the difference cor- 
relation, entries from the correlation with dark stimuli are inverted and thus carry the sign of the stimuli that causcd the spikcs.

In this experiment, we may think of spikes as "voting" for one stimulus or another. If a particular stimulus causes many spikes, it will receive a lot of votes. A stimulus that causes few spikes receives few votes. No receptive-field structure is visible in Fig. $3 A$ because there were not enough spikes to vote for any particular stimulus or set of stimuli.

Slightly later in the experiment, after 128 spikes had occurred (Fig. 3B), the discrete nature of spikes "voting" for one stimulus or another remains evident, and some stimuli have begun to get more "votes" than others. Dark stimuli located on the right side of the grid caused proportionally more spikes than dark stimuli located elsewhere, or bright stimuli located anywhere. As a consequence, the 2D spatial response profile features a barely detectable dip on the right side of the grid.

After 256 spikes had occurred (Fig. 3C), the effectiveness of dark stimuli on the right side of the grid has become prominent, and bright stimuli demonstrate a very slight tendency to cause spikes when located on the left side of the grid. In the difference surface, although the trough due to dark stimuli is now more prominent and the peak due to bright stimuli has begun to form, the surface as a whole is still very noisy. We are reluctant to draw conclusions of any type from the data at this point.

The situation improves dramatically after 512 spikes have occurred (Fig. 3D). The coherent region of excitation to bright stimuli on the left side of the grid is now obvious. On the right side of the grid, the region of preferential excitation to dark stimuli is fairly well formed and has begun to become smooth. The difference correlation now shows a prominent peak and a prominent trough reflecting the receptive-field structure. The surfaces are, however, still far from smooth.

These trends continue as more spikes accumulate. In Fig. $3 E$ (1,024 spikes), $F$ (2,048), $G(4,096)$, and $H(8,192)$, the bright and dark excitatory subregions get progressively smoother. The relative contribution of random variability (noise) in each surface continues to decrease as the relative contri- bution of each spike lessens. By the end of the experiment (Fig. $3 H$ ) the surfaces are quite smooth.

The receptive-field structure does not change dramatically as the experiment proceeds. After $\sim 512$ spikes have occurred, the surfaces exhibit all of the features evident in the final correlograms. Every reverse correlation we have run converged in this manner. Subregions do not spontaneously appear late in the run or change their shapes or spatial positions. The only important effect we have noticed is that the signal-to-noise ratio improves as the experiment progresses.

There are two additional points worth noting. First, the bright and dark excitatory subregions are well separated in space. Second, the relative amplitudes of each subregion remains the same throughout the course of the experiment (the dark excitatory subregion is slightly stronger than the bright one).

TIME SLICE. The description so far assumes coincident stimuli and spikes. This is clearly inappropriate for cells whose average visual response latency exceeds the stimulus duration. In such cases we want to examine correlations between spikes and the stimuli that precede them. In fact, it is possible that receptive-field structure changes in a systematic way over time $(1,4,29,43)$. To study spatial response profiles, we must find an appropriate interval between spikes and stimuli.

The temporal resolution of our technique is very coarse (the stimulus duration, usually $50 \mathrm{~ms}$ ), and consequently we are unable to study the time course of the responses. For the purpose of this study, we assume that $2 \mathrm{D}$ spatial response profiles do not change over time and choose for further study the temporal interval that produces the largest absolute value in the difference correlogram. This interval was always well defined, as Fig. 4 demonstrates. Here we have plotted the bright, dark, and difference correlations for stimuli preceding spikes by $0,50,100$, and $150 \mathrm{~ms}(0,1,2$, and 3 stimulus intervals). The correlation between spikes and stimuli that precede them by $50 \mathrm{~ms}$ is clearly much stronger than the others.

The temporally adjacent correlation at 100 ms shows some structure, reflecting the time course of a response sampled very coarsely. It 

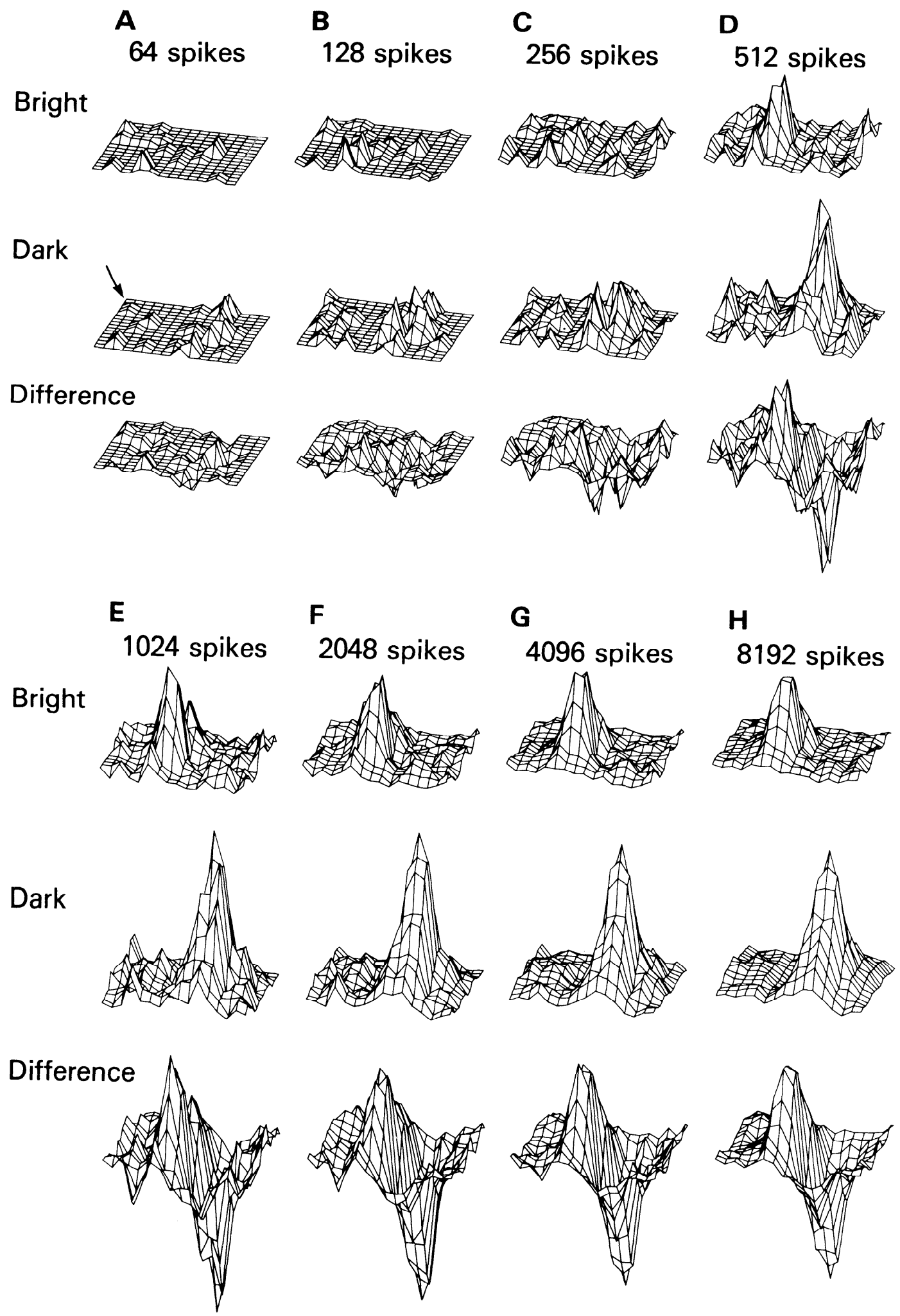
A $0 \mathrm{msec}$

Bright
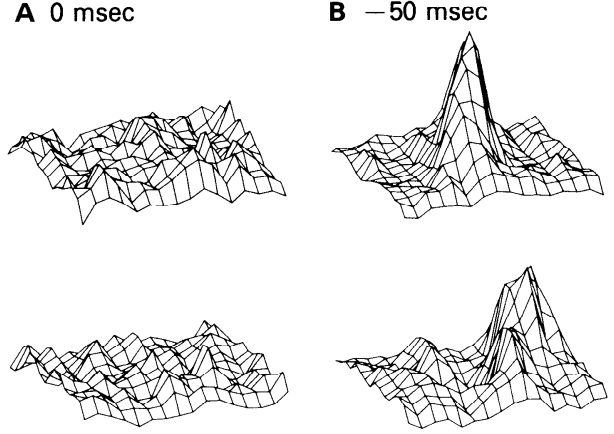

Dark
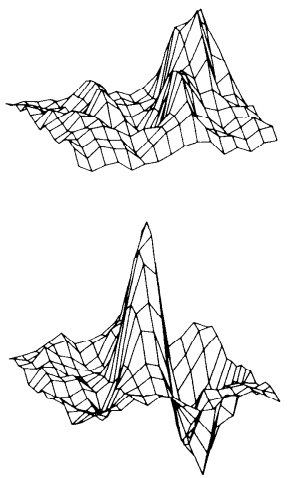

C $-100 \mathrm{msec}$
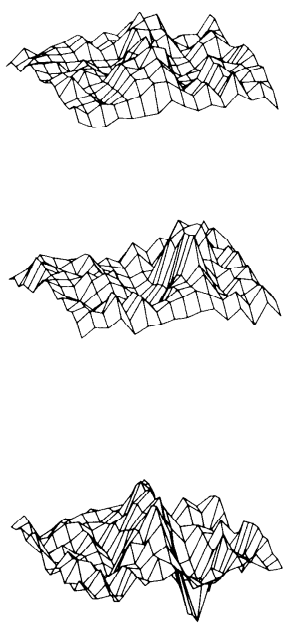

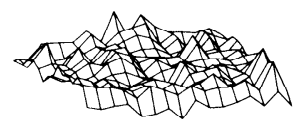

D $-150 \mathrm{msec}$
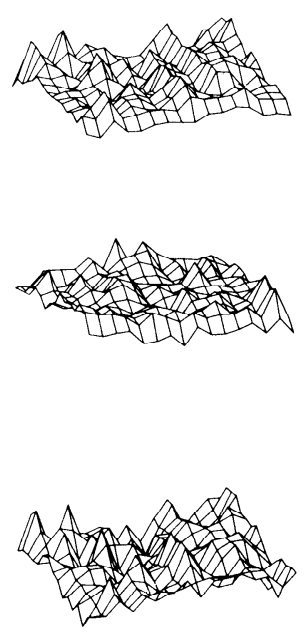

FIG. 4. Reverse correlations at different temporal intervals. $A$ : stimuli occurring coincident with spikes. No receptive-field structure is evident. $B$ : stimuli preceding spikes by $50 \mathrm{~ms}$. The receptive-field structure is prominent and consists of a central subregion excited by bright stimuli, with 2 weaker flanking subregions excited by dark stimuli. $C$ : stimuli preceding spikes by $100 \mathrm{~ms}$. The same receptive-field structure as $B$ is evident, only much weaker. $D$ : stimuli preceding spikes by $150 \mathrm{~ms}$. No receptive-field structure is evident. The temporal interval producing the largest correlation $(B)$ was chosen for further study.

if evident that the response magnitude is smaller in this correlation but that the form of the response profile is about the same (one bright excitatory subregion, with two somewhat weaker dark excitatory subregions flanking it). The correlations with coincident stimuli and stimuli preceding spikes by 150 ms show no evident structure. As in this case, nearly every cell exhibited a distinct peak in the reverse correlation.

REPRODUCIBILITY. The 2D spatial response profiles obtained with the reverse correlation method are independent of small changes in stimulus parameters. The reverse correlation program had 12 parameters under user control. We were not able to systematically vary them individually, let alone jointly, to study the effects each had. Nevertheless, in many repeated acquisitions of spatial response profiles from individual cells, the surfaces were unaffected by reasonable variation in stimulus parameters.

The contour plots of Fig. 5 provide five examples. The general features of the measured 2D spatial response profiles were largely unaffected by varying the orientation of the stimulus grid (Fig. 5, $A, C, D$ ), size of the explored region and stimulus size (Fig. 5, $B-E)$, size of the stimulus within the grid (Fig. $5 D$ ), and stimulus duration (Fig. $5 E$ ). It is especially noteworthy that the results were invariant across rather large changes in ori-

FIG. 3. Growth and convergence of the reverse correlation. Each trio of surfaces illustrates the correlation between the spike train and bright stimuli (top row), dark stimuli (middle row), and the difference of these 2, the two-dimensional spatial response profile (bottom row). $A-D$ : drawn with the same $z$-axis (response axis) scale. $A$ : 64 spikes, 136 stimuli. An example of the effect of a single spike is marked with an arrow in the correlation with dark stimuli. $B$ : 128 spikes, 264 stimuli. $C$ : 256 spikes, 504 stimuli. $D: 512$ spikes, 1,250 stimuli. $E-H$ have been drawn with different $z$-axis scales to keep the graphs on the page. $E$ : 1,024 spikes, 2,495 stimuli, $2 / 3$ the scale of $D$. F : 2,048 spikes, 4,986 stimuli, $1 / 2$ the scale of $E$. $G: \quad 4,096$ spikes, 9,739 stumuli, $1 / 2$ the scale of $F$. $H: \quad 8,192$ spikes, 18,296 stimuli, $1 / 2$ the scale of $G$. The receptive field does not change its structure as the experiment proceeds; the signal-tonoise ratio simply increases. 


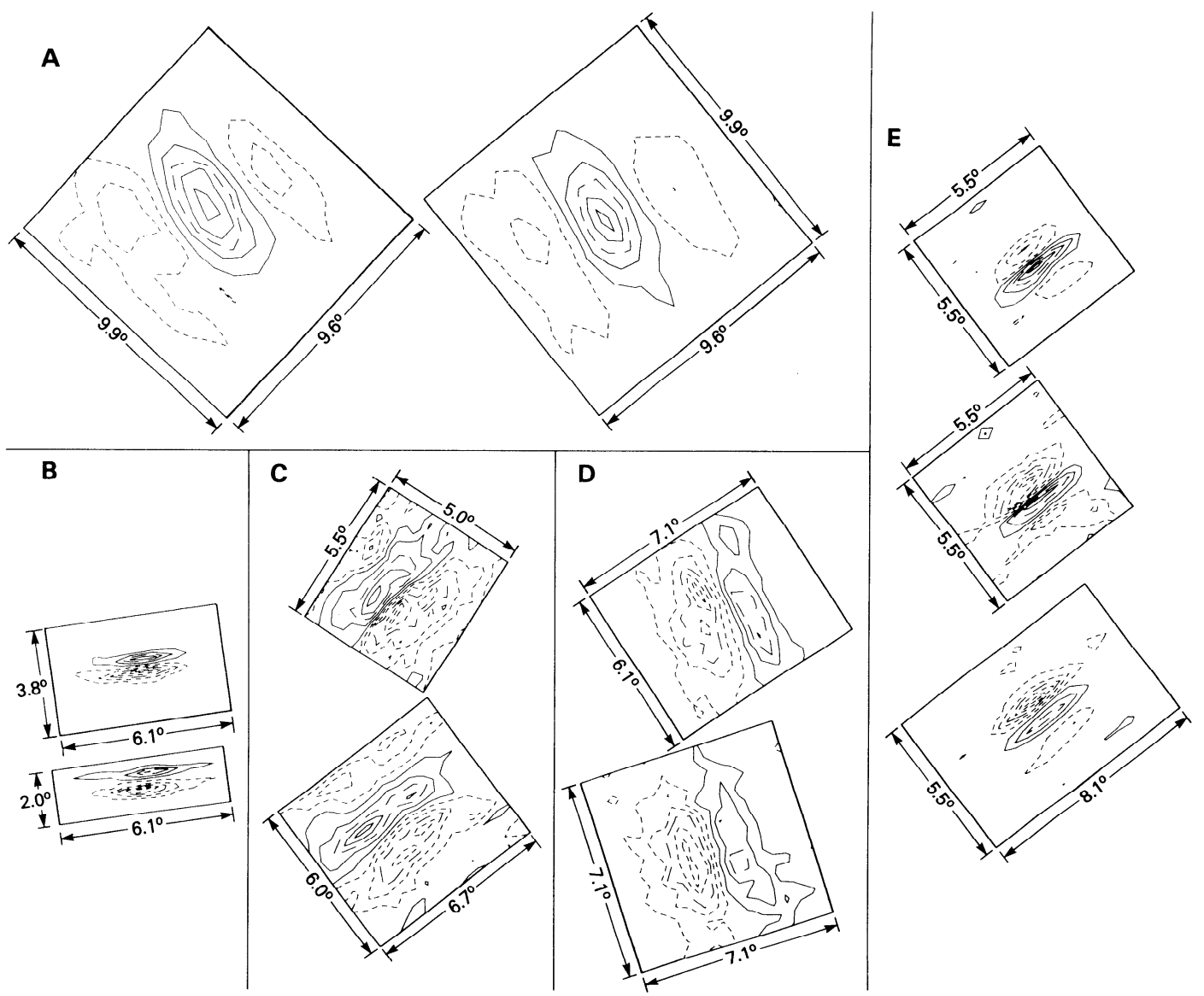

FIG. 5. The reverse correlation yields reproducible results independent of small changes in stimulus parameters. Each part illustrates multiple trials on single receptive fields. In each case, the two-dimensional spatial response profile is both reproducible and insensitive to small changes in stimulus parameters. The contour plots are drawn to a common spatial scale and in the correct relative orientations. Reverse correlation stimulus parameters differ in the following ways. $A$ : grid orientation. The axes of the grid are rotated by $7^{\circ} . B:$ absolute stimulus size. The stimulus widths differ by about a factor of 2. C: both grid orientation (difference of $20^{\circ}$ ) and absolute stimulus size (factors of $3 / 4$ and $4 / 5$ in the width and length directions). $D$ : grid orientation $\left(15^{\circ}\right.$ ), absolute stimulus size (factor of 2.5 in the length direction), and stimulus size within the grid. The first trial was run with $1 \times 3$ grid unit stimuli, the second with $1 \times 1$ grid unit stimuli. $E$ : stimulus duration and stimulus size for 3 trials on a single cell. The grid orientation in each case is the same. The 1 st trial was run with $50-\mathrm{ms}$ stimuli, the 2 nd and 3 rd with $100-$ ms stimuli. The stimulus sizes in the first 2 trials were the same and differ from the 3rd trial by a factor of 1.5 in the length direction.

entation of the grid on which stimuli were presented. This is most evident in Fig. $5 C$ where the grid orientation differed by $20^{\circ}$ on two successive measurements. The differences in receptive-field structure evident in each case illustrated in Fig. 5 may be attributed to random variability in the response.

Presumably, if certain stimulus parameters were changed drastically, different spatial response profiles would result. It is likely, for example, that very large stimuli would obscure the subregions. Nevertheless, infor- mal observations like those in Fig. 5 convince us that variation of stimulus parameters within reasonable bounds do not change the 2D spatial response profiles of simple cells.

Part II. 2D spatial response: profiles of simple cells

THE DISTRIBUTION OF EXCITABILITY IN SPACE. The 2D spatial response profiles of simple cells observed in this study consisted of two or three subregions alternating in sign 
across the width of the receptive field. Although the details of receptive-field structure varied widely in our population, we first consider characteristics exhibited by all the response profiles.

1) Adjacent subregions do not overlap. This is best seen in contour plots such as those of Fig. 6, $A$ and $B$, where the bright and dark stimulus correlations are normalized and presented separately. Because the stimuli have finite widths, one might expect stimuli falling on the edge of a subregion to encroach slightly on an adjacent subregion of opposite polarity. The response profile in Fig. $6 \mathrm{C}$ illustrates the most extensive example of subregion overlap we have observed. Similarly, we have not observed profiles with unresponsive regions intercalated between subregions of opposite sign.

2) Each subregion has a single well-defined extremum. This is evident from the contour diagrams shown in Figs. 6 and 7 and throughout the results. Occasional exceptions such as the bright excitatory subregion
A

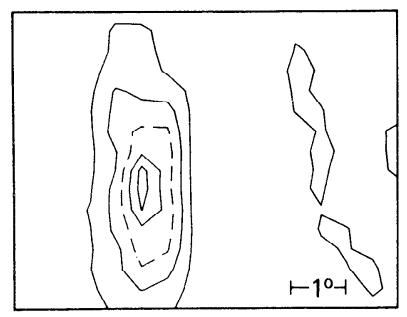

Bright

Dark
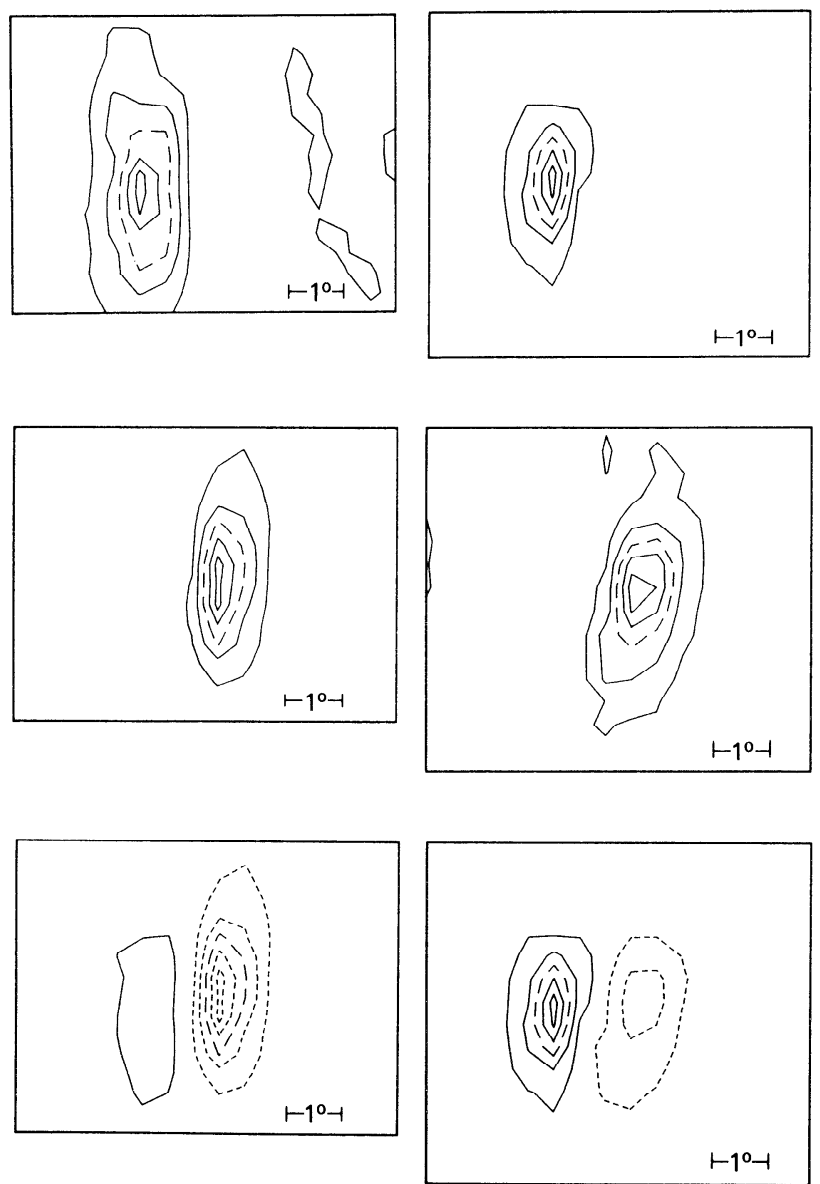

C
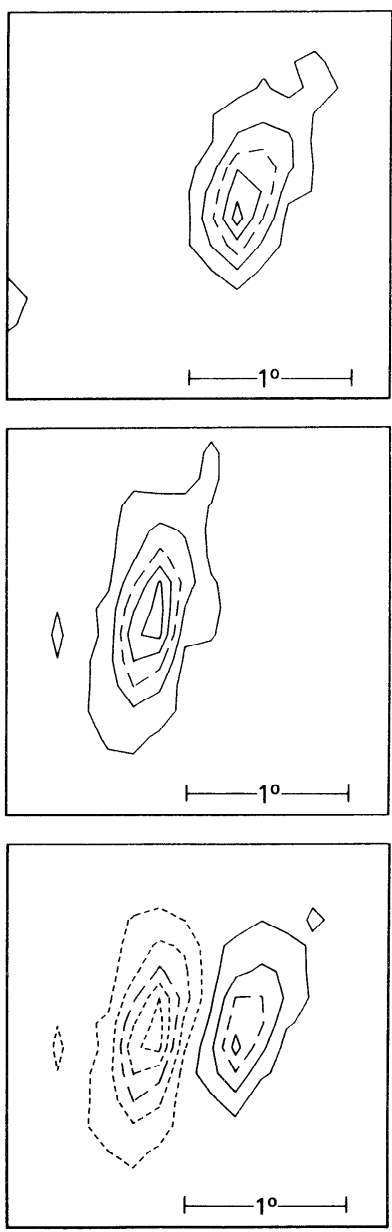

FIG. 6. Simple receptive fields consist of spatially segregated subregions. The correlation with bright stimuli, dark stimuli, and the difference of these are illustrated for 3 example cells. The bright, dark, and difference correlations have been normalized separately in each case. $A$ : a receptive field with a strong response to dark stimuli and a weaker response to bright stimuli. No spatial overlap of the 2 subregions is apparent. $B$ : a reccptive field with a strong response to bright stimuli and a weaker response to dark stimuli. No spatial overlap of the 2 subregions is apparent. $C$ : in the most extensive example of subregion overlap observed, the subregions overlap by $<20 \%$ of their respective widths. 
on the left in Fig. $7 C$ are reasonably attributed to noise.

3) The transition from one subregion to another is smooth and continuous. The 2D spatial response profile illustrated in Fig. $7 C$ provides a good example. In the grid surface, three lines running parallel to the long axis of the field are visible on the right side of the bright excitatory subregion. Because samples taken along these three rows of the grid were independent of one another, this shows that the extrema of the two subregions were displaced in space and that the transition between the two occurred smoothly. In the contour plot, it can be seen that the transition from peak to trough occupies about $1 / 3$ of the entire width of the grid. All of the examples in Fig. 7 behave in this way.

4) The subregions decayed smoothly into the area surrounding the receptive field. Figure $7 \mathrm{~B}$ provides a typical example. In the grid surface, both the bright excitatory subregion and the dark excitatory subregion decay smoothly from their respective extrema as one moves forward. In the contour plot, it is evident that this decay is roughly symmetrical in both directions along the length axes of the individual subregions. Because decay into the surrounding noise is also evident in the width direction, the determination of receptive-field dimensions and area is clearly a matter of definition.

5) The subregions are elongated and parallel to one another. These features are conspicuous in all of the examples in Fig. 7 as well as those shown throughout this and the third paper of this series. The length-to-width ratios of individual excitatory subregions (measured at the $1 / e$ contour level) ranged from 1.7 to 12.0 . We have observed no square, rectangular, or circular subregions, or any unambiguous example of nonparallel subregions.

The most obvious way in which the $2 \mathrm{D}$ spatial response profiles of simple cells varied is the number of subregions, their relative amplitudes, and their relative positions on the plane. The basic patterns observed in this study, illustrated in Fig. 7, have been demonstrated many times in earlier 1D studies of striate cortex $(9,27,33,34,40)$. We have previously designated the cell types of Fig. 7 as $S-2$ and $S-3$, referring to the number of excitatory subregions (34).
There is considerable variation in the dimensions of individual excitatory subregions comprising the response profiles of simple cells. Several factors contributed to this variation including the range of eccentricities of the receptive fields studied. However, a large source of this variation arose within the response profiles of individual simple cells. In response profiles with three excitatory subregions, for example, it was often the case that the flanking regions were shorter than the central region. Proportionately, the lengths of subregions comprising a single response profile show more variation than do the widths. Our sample of cells is too small to draw any meaningful conclusions regarding cell-to-cell variation in subregion size.

The features described above are evident in other 2D spatial response profiles illustrated in this paper and the third paper of the series (18).

SYMMETRY. Previous investigators have generally recognized two or more classes of simple cells based on the number of excitatory subregions in their receptive fields. Receptive fields with an odd number of subregions are frequently considered to be even symmetric, whereas those with an even number of subregions are considered odd symmetric. Even and odd symmetry imply that specific quantitative relationships exist between various parts of the receptive fields

$$
\begin{array}{ll}
\text { even symmetry: } & w(x)=w(-x) \\
\text { odd symmetry: } & w(x)=-w(-x)
\end{array}
$$

where $w(x)$ denotes the $1 \mathrm{D}$ width axis response profile, and $x=0$ is taken as the center of the receptive field. If $2 \mathrm{D}$ spatial response profiles are simply elongated versions of the 1D spatial response profiles these relationships generalize to

$$
\begin{aligned}
\text { even symmetry: } & \mathrm{r}(x, y)=\mathrm{r}(-x, y) \\
\text { odd symmetry: } & \mathrm{r}(x, y)=-\mathrm{r}(-x, y)
\end{aligned}
$$

where $\mathrm{r}(x, y)$ denotes the 2D spatial response profile, $x=y=0$ is the center of the receptive ficld, and the coordinate system is aligned with the axis of elongation. We note that this is only one of the possible $2 \mathrm{D}$ symmetry relationships.

There is, however, no a priori reason to expect response profiles to be limited to even and odd symmetric forms. In fact, most of 
A
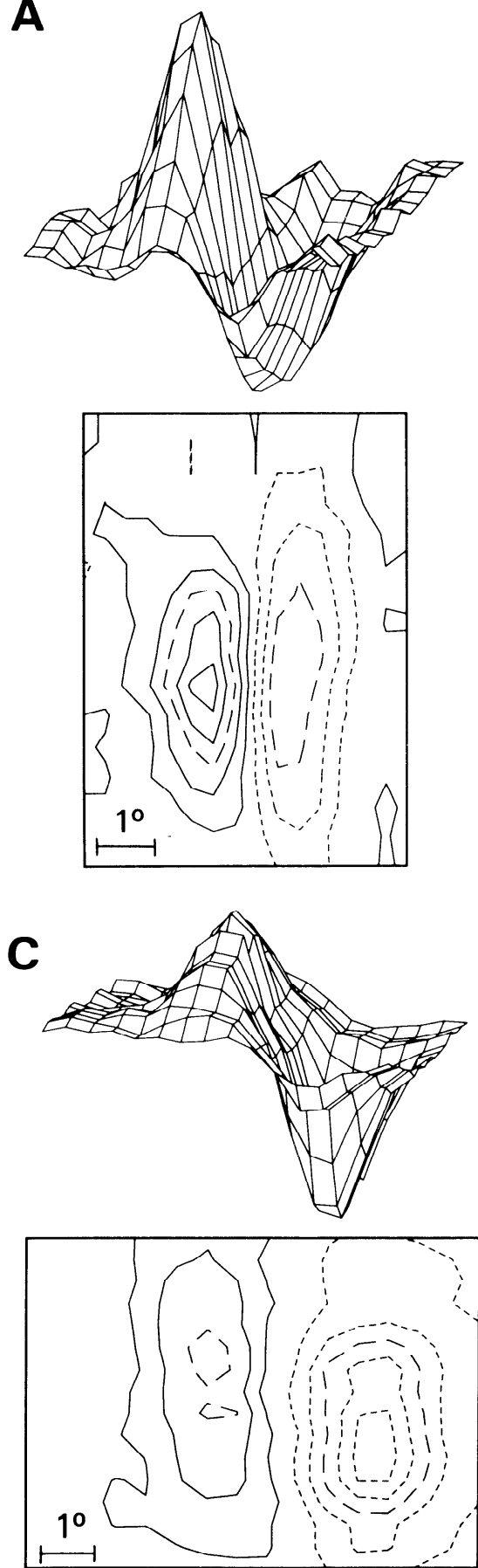

B
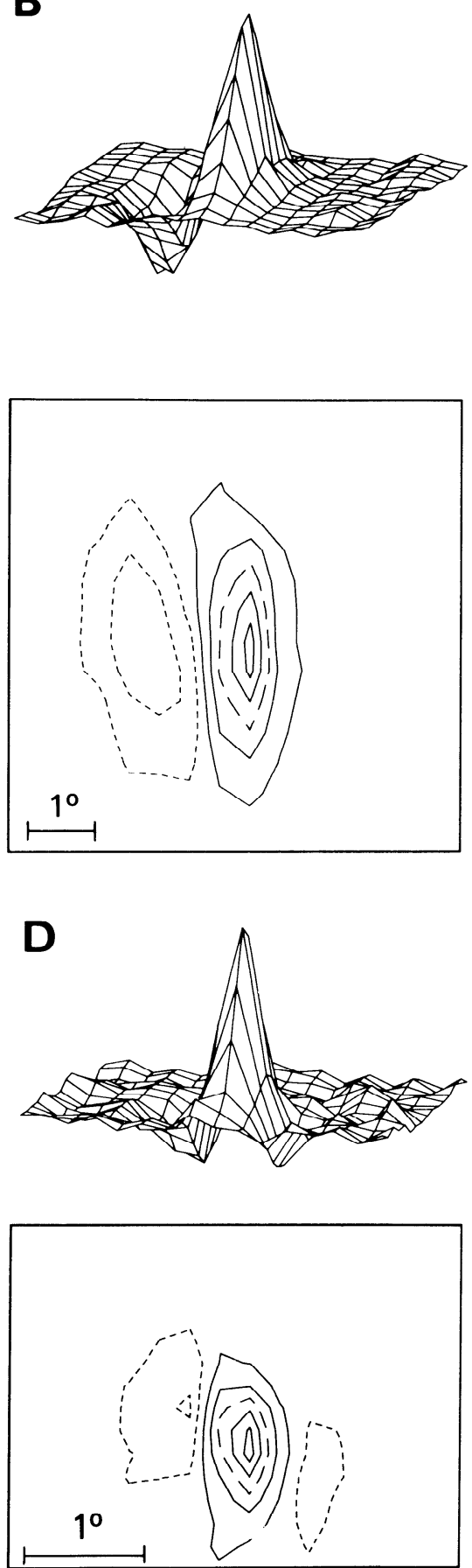

FIG. 7. The two-dimensional spatial response profiles of 4 typical simple receptive fields illustrate variability in the relative sizes, shapes, and placements of individual subregions. $A$ : the bright excitatory subregion is stronger than the dark excitatory subregion, which is somewhat longer. $B$ : the bright excitatory subregion is stronger than the dark excitatory subregion, but the sizes and shapes of the 2 subregions are about the same. $C$ : the dark excitatory subregion is stronger than the bright excitatory subregion, and the peaks of the 2 are not mirror symmetric about the receptive-field zero crossing. $D$ : a simple receptive field with 3 excitatory subregions, 1 to bright stimuli and 2 to dark stimuli. The central bright excitatory subregion is the strongest, and the 2 flanking subregions are approximately the same strength. Note the relative placements of the individual subregions. 
the 2D spatial response profiles in our sample did not exhibit perfect even or odd symmetry. Figure 8 illustrates four examples. The two subregions of the response profiles in Fig. $8 A$ are virtually identical in amplitude, and we may conclude that this response profile is odd symmetric. The bright excitatory subregion in Fig. $8 B$ is slightly stronger than the dark excitatory subregion; although this response profile is close to odd symmetry, it is not odd symmetric. The response profiles in Fig. 8, $C$ and $D$, illustrates extreme deviations from odd symmetry as a consequence of the unequal amplitudes of the two subregions.

However, one could argue that this spatial asymmetry results from low resolution sampling along the width axis of the receptive field. Because the number of samples was 16 , this is unlikely to be a problem for the loworder receptive fields in this population ( 2 or 3 excitatory subregions). In addition, however, some cells were studied with higher resolution $1 \mathrm{D}$ reverse correlation. This procedure is identical to the $2 \mathrm{D}$ reverse correlation, except the number of samples in the length direction has been collapsed to one, and the stimulus length was increased to the full grid length. It was never observed that the symmetry or lack thereof changed significantly with doubling or even quadrupling the number of samples in the width dimension. Examples of width direction 1D spatial response profiles obtained with a 32 position 1D reverse correlation are shown in Fig. 9 (see also Table 2). The profiles illustrated in Fig. 9, $A$ and $C$, are close to even and odd symmetric, respectively. But it is evident that the response profiles illustrated in Fig. 9, $B$ and $D$, are neither even nor odd symmetric despite the higher spatial resolution.

SEPARABILITY. As noted in the introduction, an underlying assumption of a $1 \mathrm{D}$ (length/width) approach to the 2D structure of simple receptive fields is that the fields are Cartesian separable. That is, the receptive field is the product of some function along the width axis and some other function along the length axis

$$
\mathrm{r}(x, y)=w(x) \cdot l(y)
$$

where $\mathrm{r}(x, y)$ denotes the 2D spatial response profile, $w(x)$ the width axis response profile,

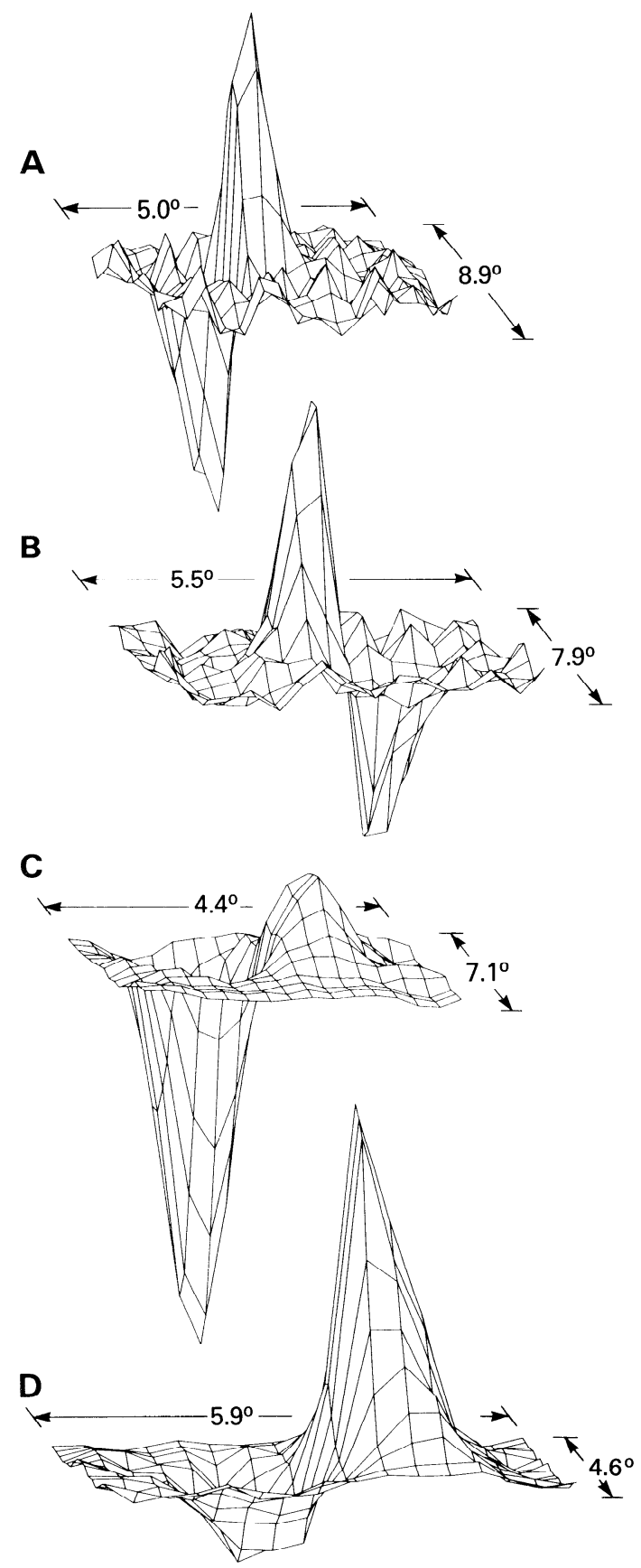

FIG. 8. Simple receptive fields are not necessarily spatially symmetric. $A$ : an odd-symmetric response profile. The bright excitatory subregion and the dark excitatory subregion are the same strength. $B$ : a response profile close to odd symmetry. The bright excitatory subregion is slightly stronger than the dark excitatory subregion. $C$ and $D$ : illustrations of highly asymmetric response profiles. In $C$, the dark excitatory subregion is much stronger than the bright excitatory subregion, whereas in $D$ the converse is true. 

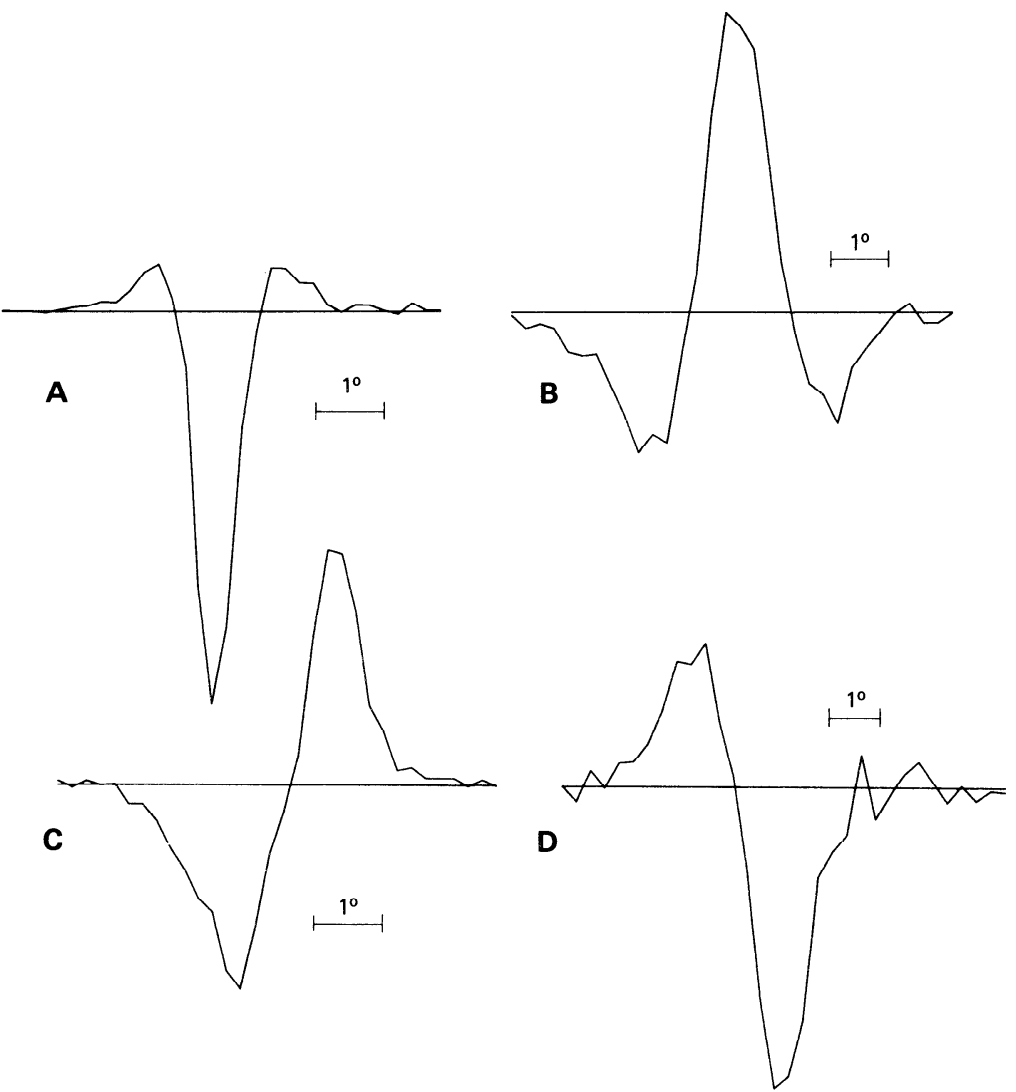

FIG. 9. One-dimensional (1D) response profiles obtained with a $1 \mathrm{D}$ reverse correlation program with 32-position spatial resolution confirm that simple receptive fields are found in asymmetric forms. $A$ : an even-symmetric 1D response profile. $B:$ a response profile close to even symmetry. $C:$ an odd-symmetric response profile. $D$ : a response profile close to odd symmetry. The data in $A-C$ were obtained with 50 -ms stimuli. Noteworthy is the fact that the data in $D$ were collected with 20 -ms stimuli. The stimulus parameters for each experiment are given in Table 2.

and $1(y)$ is the length axis response profile. Since $\mathrm{r}(x, y)$ is now available, it is possible to evaluate this assumption.
If $2 \mathrm{D}$ spatial response profiles are Cartesian separable, we can expect to make certain observations in all cases. First, linear sections

TABLE 2. Stimulus parameters for each experiment shown in Fig. 9

\begin{tabular}{|c|c|c|c|c|c|}
\hline \multirow[b]{3}{*}{ Cell } & \multirow[b]{3}{*}{$\begin{array}{l}\text { No. of } \\
\text { Spikes }\end{array}$} & \multicolumn{4}{|c|}{ Stimuli } \\
\hline & & \multirow[b]{2}{*}{ No. } & \multirow[b]{2}{*}{$\begin{array}{c}\text { Time, } \\
\mathrm{ms}\end{array}$} & Sizc, deg & \multirow[b]{2}{*}{$\begin{array}{c}\text { Orientation, } \\
\text { deg }\end{array}$} \\
\hline & & & & w & \\
\hline$A$ & 5,854 & 12,355 & 50 & $0.2 / 7.2$ & 166 \\
\hline$B$ & 4,660 & 23,746 & 50 & $0.3 / 6.2$ & 118 \\
\hline$C$ & 8,192 & 26,798 & 50 & $0.2 / 6.0$ & 35 \\
\hline$D$ & 2,060 & 24,653 & 20 & $0.3 / 7.2$ & 98 \\
\hline
\end{tabular}

Profiles are identified as in Fig. 9. Stimulus parameters are given in terms of the number of stimuli delivered (no.), the temporal duration of each stimulus (time), the width (w) and length (1) of individual stimuli in degrees of visual angle, and the orientation in degrees counter clockwise from horizontal. 
taken through the response profile parallel to the width axis will be identical except for a scale factor. Second, linear sections taken through the response profile parallel to the length axis will be identical except for a scale factor. Third, if we multiply (vector product) the average of the normalized width direction sections (which are identical by expectation 1) by the average of the normalized length direction sections (which are identical by expectation 2), we should recover the $2 \mathrm{D}$ response profile exactly. If Cartesian separability is the rule, this result would allow 2D spatial response profiles to be generated from two 1D profiles taken along the width and length axes. To test these expectations, we subjected 2D spatial response profiles to the analysis described below.

The 2D spatial response profile illustrated in Fig. $10 \mathrm{~A}$ was sectioned parallel to the width axis by taking the data from each row in the grid whose maximum absolute value cxceeded $1 / e$ of the maximum absolute value over the whole surface. These sections are graphed, one on top of another, below the contour plot (Fig. 10B). The thresholding operation was performed to eliminate sections that may reside outside of the receptive field and would not be expected to contribute to the receptive-field structure. In the graph immediately below (Fig. 10C), the sections have been normalized so that their maximum absolute values are equal. Because they are virtually identical, expectation $l$ is satisfied. The average of these normalized sections is graphed at the bottom (Fig. $10 D)$. We will use this average of sections to reconstruct the $2 \mathrm{D}$ receptive-field profile from width and length sections through the field.

The absolute values of sections taken parallel to the length axis of the response profile are illustrated immediately to the right of the original data (Fig. 10E), following the $1 / e$ thresholding operation as bcfore. The absolute value is taken, since the sign of the response changes at the transition from a bright excitatory subregion to a dark excitatory subregion. (The width direction sections carry the sign.) Again, the normalized sections (Fig. 10F) are virtually identical, thus satisfying expectation 2 . At the far right (Fig. 10G) the normalized sections have been averaged as for the width direction sections.
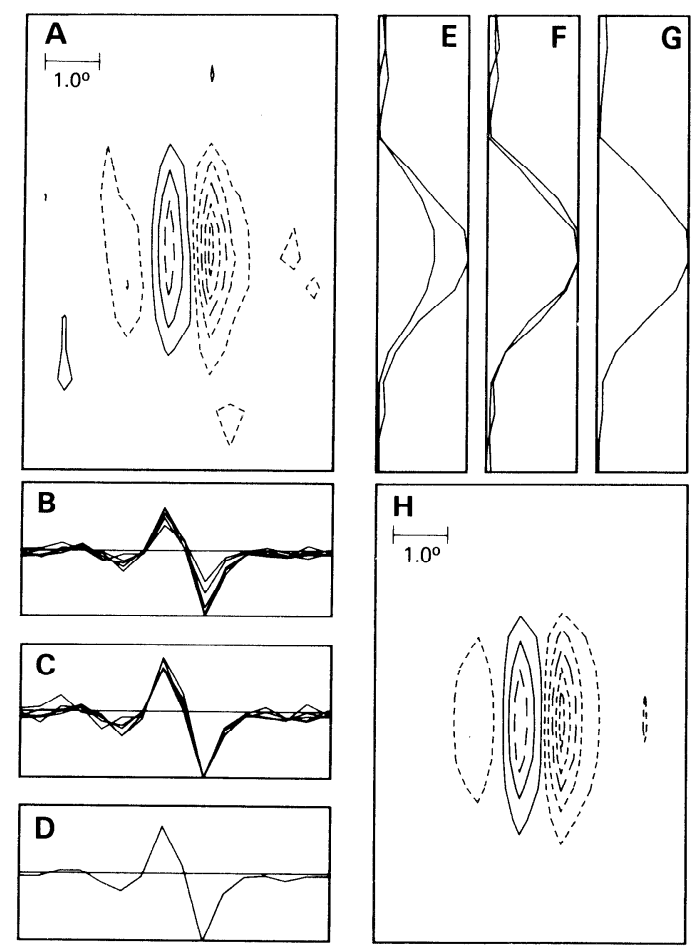

FIG. 10. A Cartesian separable simple receptive field. $A$ : the original two-dimensional (2D) spatial response profile. $B$ : sections parallel to the width axis whose individual extrema exceed $1 / e$ of the global extremum. $C$ : the sections in $B$ have been normalized. They are essentially identical. $D$ : the average of the sections in $C$. $E$ : the absolute values of 2 sections parallel to the length axis whose extrema exceed $1 / e$ of the global extremum. $F$ : 2 normalized sections in $E$ that are essentially identical. $G$ : The average of the sections in $F$. $H$ : The $2 \mathrm{D}$ spatial response profile is reconstructed under the assumption of Cartesian separability using the average width axis section $(D)$ and the average length axis section $(G)$. The original response profile is reconstructed with good fidelity. Thus the response profile is Cartesian separable.

Finally, the average width section and the average length section were multiplied to produce a reconstructed $2 \mathrm{D}$ receptive-field profilc, illustrated in Fig. $10 \mathrm{H}$. This contour plot should be compared with the contour plot of the original data (Fig. 10A). In this case, the reconstructed $2 \mathrm{D}$ response profile is very similar to the original data. Thus expectation 3 is satisfied. The reconstructed field is somewhat smoother than the original, reflecting the averaging operation. The fidelity of the reconstruction convinces us that the original 2D spatial response profile is the 
product of independent length and width direction response profiles. In this case, the assumption of Cartesian separability is correct.

We now consider a $2 \mathrm{D}$ spatial response profile that is not Cartesian separable. The response profile illustrated in Fig. $11 \mathrm{~A}$ was sectioned parallel to the width axis following the same thresholding criteria as before. The resulting sections are illustrated in Fig. $11 B$. In the graph immediately below (Fig. 11C), all of the sections have been normalized so that their extreme values are equal. In this case, the sections are not the same. Although the extrema of each section occur in the same place, the relative amplitudes change. Specifically, the left-hand subregion grows weaker, and the right-hand subregion stronger, as one moves top to bottom across the original response profile. Thus expectation $l$ is not satisfied. The average of these sections is graphed at the bottom (Fig. 11D).

Sections taken parallel to the length axis of the receptive field are graphed immediately to the right of the original data (Fig. $11 E$ ), using the absolute value of the response as before. Again, the normalized sections (Fig. $11 F$ ) are not the same. Specifically, the peaks shift from top to bottom as one moves left to right across the original response profile. Consequently, expectation 2 is not satisficd. The average length direction section is plotted at the far right (Fig. 11G).
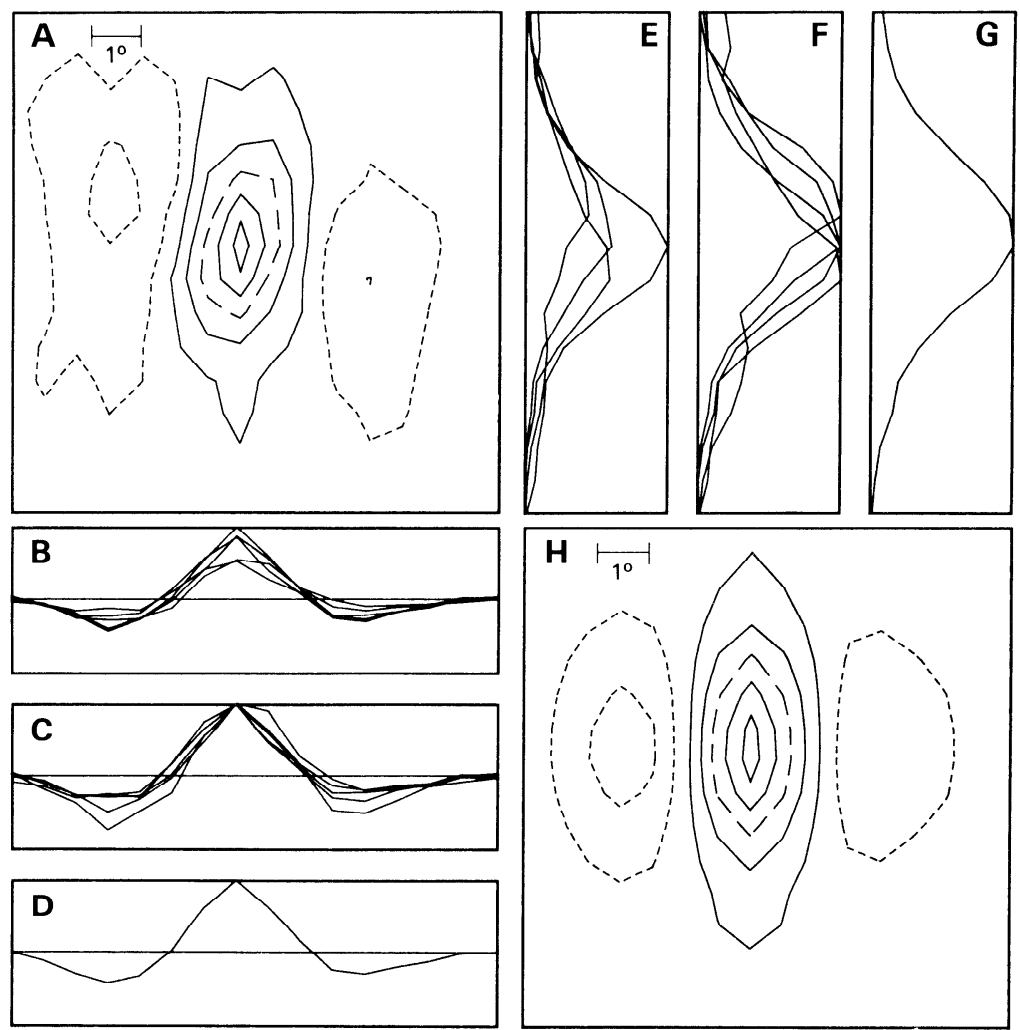

FIG. 11. A simple receptive field that is not Cartesian separable. $A$ : the original two-dimensional (2D) spatial response profile. $B$ : sections parallel to the width axis whose individual extrema exceed 1/e of the global extremum. $C:$ the sections in $B$ that have been normalized. The extrema of each section occur in the same place, but the amplitudes are not the same. $D$ : the average of the sections in $C . E$ : the absolute values of sections parallel to the length axis whose extrema exceed $1 / e$ of the global extremum. $F$ : the sections in $E$ that have been normalized. Although the shapes of these sections are similar, the peaks occur in different places. $G$ : the average of the sections in $F . H$ : the 2D spatial response profile is reconstructed under the assumption of Cartesian separability using the average width axis section $(D)$ and the average length axis section $(G)$. The reconstruction retains many of the features of the original response profile, but the relative positions of the subregions are lost. The original $2 \mathrm{D}$ spatial response profile is not Cartesian separable. 
Finally, the average width section and the average length section were multiplied to produce a reconstructed 2D spatial response profile, shown in Fig. $11 \mathrm{H}$. This contour plot should be compared with the original data (Fig. 11A). In this case, the reconstructed response profile is different from the original data. Specifically, the flanking dark excitatory subregions are shifted from their origi- nal positions and are now aligned along the width axis. Thus expectation 3 is not satisfied. Because these data failed to satisfy all our expectations, we conclude that this $2 \mathrm{D}$ spatial response profile is not Cartesian separable.

Using these methods we found that about half of the 2D spatial response profiles in our population were not Cartesian separable.
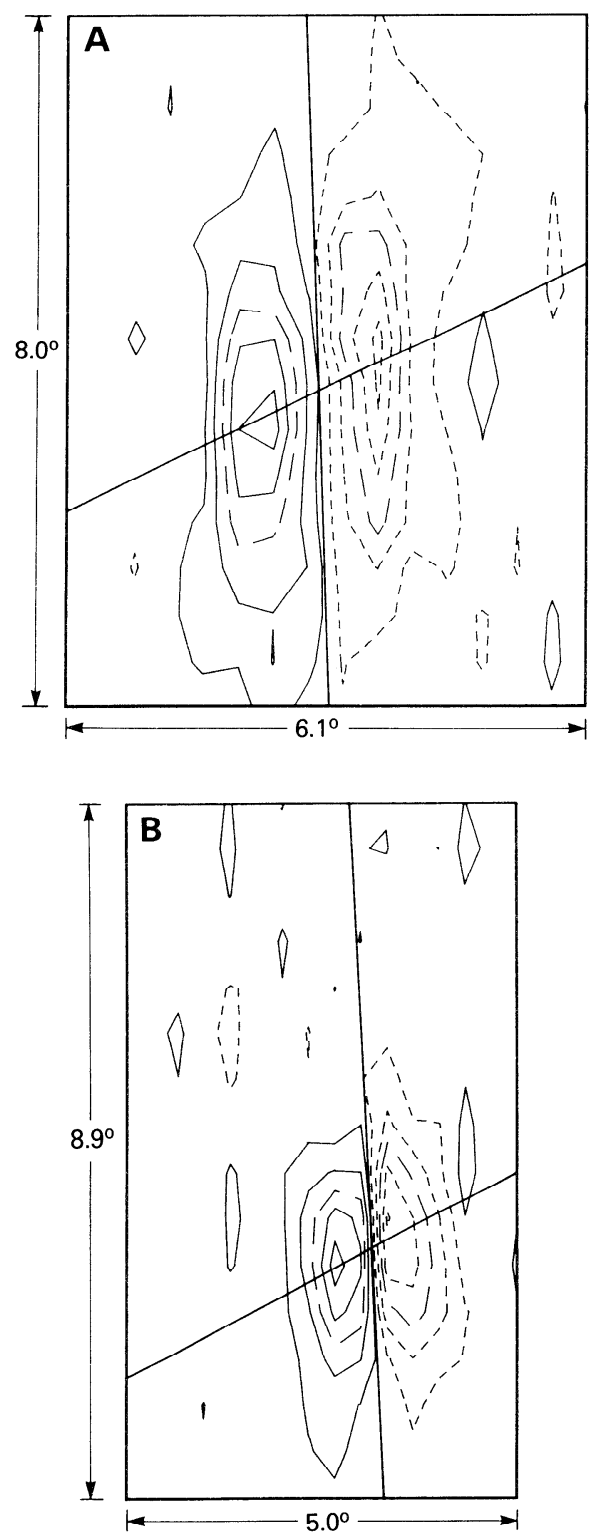
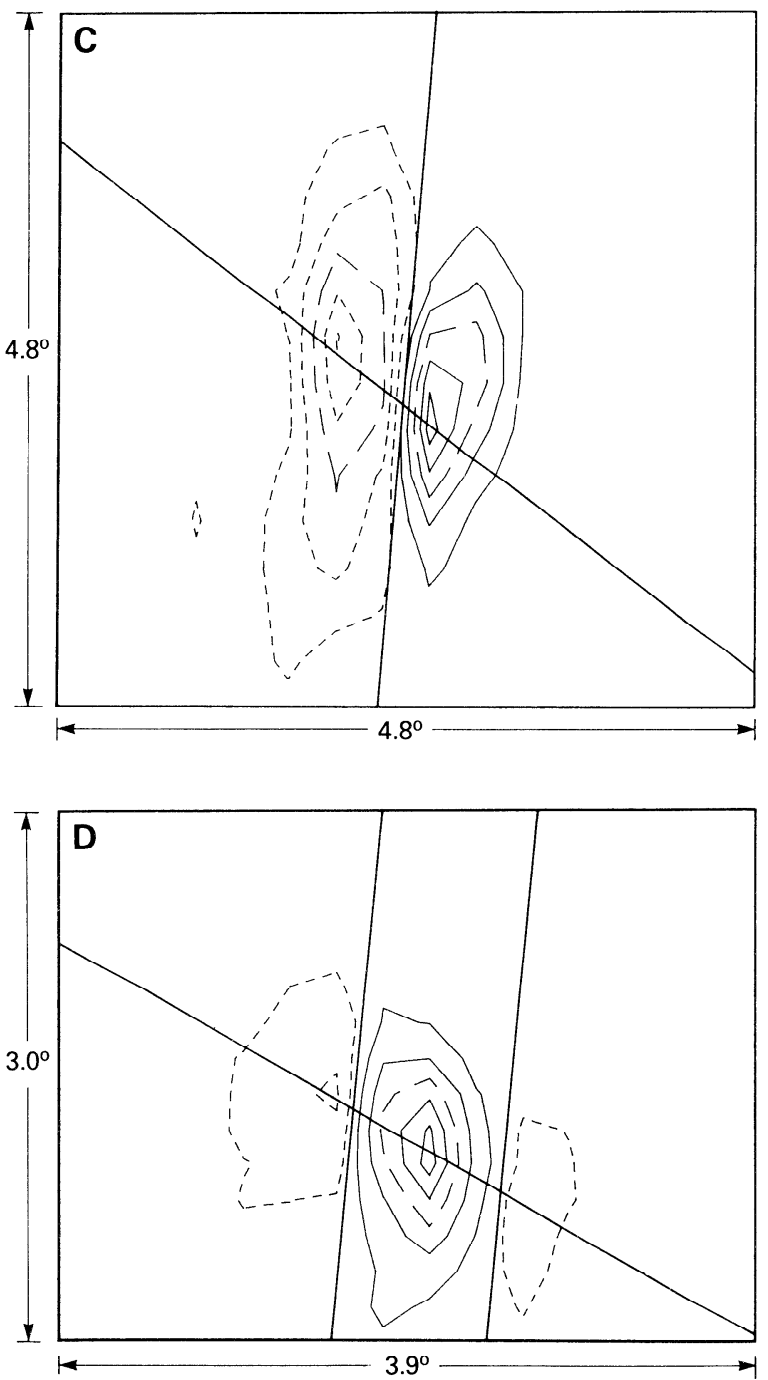

FIG. 12. Simple receptive fields that are not Cartesian separable are common. If the response profiles were Cartesian separable, lines drawn along the receptive-field zero crossings would be perpendicular to lines connecting the receptive-field extrema. $A-D$ illustrate 4 examples where this test fails. 
There are many ways in which a response profile can fail to be Cartesian separable, but in every case it appeared to arise from a single phenomenon: individual subregions were displaced from their expected positions along an axis parallel to their long dimensions.

Figure 12 illustrates additional examples in a different format. Lines superimposed on each contour plot were drawn by eye 1) along the zero crossings between excitatory subregions of opposite polarity, and 2) connecting the extrema of each subregion. In Cartesian separable response profiles, such lines are perpendicular. We can use this fact to test response profiles for Cartesian separability even in cases where the sampling grid is not perfectly aligned with the long axis of the subregions. This test, although less rigorous than that used previously, may be applied to contour plots at a glance. It is apparent that each of the response profiles illustrated in Fig. 12 fail this test and would fail the other tests for Cartesian separability in the same way as the response profile of Fig. 11. Note also that each of the response profiles in Fig. 12 fails to satisfy even symmetry along the length axis.

We have examined Cartesian separability in simple receptive fields at a binary (yes or no) level. We must defer the measurement of the extent to which a response profilc is or is not Cartesian separable until we have developed a good model of overall receptive-field structure (18).

\section{DISCUSSION}

\section{D spatial structure of simple receptive fields}

We have provided a quantitative description of the organization of simple receptive fields in 2D of space. Many of our results are in accord with earlier $1 \mathrm{D}$ studies $(9,12,17$, 21, 26, 27, 29, 33, 34, 40). Spatial response profiles of simple cells consist of multiple elongated parallel subregions alternately driven by stimuli of opposite contrast. We observe virtually no overlap between adjacent subregions. Subregions decay smoothly in all directions from their individual extrema; those on the edge of the receptive field blend gradually into the noise making the measurement of their absolute sizes a matter of definition. The widths and lengths of individual subregions (measured by hand at the $1 / e$ contour) may vary within single receptive fields. The degree of variability depends on the spatial symmetry of the receptive field and the number of subregions included.

In previous reports from this laboratory $(29,34)$ we have demonstrated the existence of simple cells with one excitatory subregion in their receptive fields. The evidence in favor of this classification was the existence of bright and/or dark inhibitory subregions in the receptive field, which are not observed in complex cells. In the present study we have not included these receptive fields in our population, since we had no way of unambiguously verifying the existence of these subregions.

Furthermore, we (30) and others (20, 27) have observed simple receptive fields with more than three spatially discrete subregions. In a previous study of nearly 200 simple cells, wc observed $\sim 10 \%$ with more than three subregions. Our failure to find these receptive fields in this study probably results from several factors. First, our present version of the 2D spatial reverse correlation program is limited to 16 samples in each direction: we previously argued that a large number of samples was necessary to observe more than three subregions. Second, our previous study was restricted to the central $10^{\circ}$ of the visual field, whereas the present sample is distributed largely beyond $5^{\circ}$ of eccentricity. It is possible that there are proportionally fewer periodic receptive fields at this eccentricity. Further, the present study involves only 36 cells, of which at most 4 would be expected to have more than 3 subregions, even if the fraction of periodic receptive fields were the same in more eccentric locations in the visual field. We expect that if the resolution of our technique were improved and we sampled more centrally in the visual field we would observe simple receptive fields with many subregions. Finally, we recognize that our stimuli are small and of low power and may not excite very weak subregions visible during relatively short acquisition periods. However, preliminary comparisons with receptive-field estimates derived from more conventional stimulus ensembles (slowly moving long bars) suggest that we are not systematically underestimating either re- 
ceptive-field width or the number of included subregions.

\section{Separability and symmetry}

Our results differ most notably from those of earlier workers on the issues of spatial symmetry and Cartesian separability. The usual assumption that simple receptive fields are found in even or odd symmetric forms $(22,24,27)$ was not verified in this study. We found very few cells whose receptive fields exhibited perfect even or odd symmetry. In the last paper of this series, we will establish a means for measuring spatial phase angle and will show that this variable is uniformly represented in our small population of simple cells (18). This result is in complete agreement with the 1D study of Field and Tolhurst (10). Thus, although it is computationally convenient and mathematically appealing to imagine only even and odd symmetric simple receptive fields, this is clearly not accurate.

Asymmetric receptive fields are consistent with the observation of Pollen and Ronner (36) that simultaneously recorded simple cells in cat striate cortex are found in quadrature relative phase. We have not systematically examined the response profiles of simultaneously recorded cells, but we have no reason to doubt their result. It is noteworthy that pairs of cells with cospatial receptive fields and quadrature relative phase angles could be used to represent stimulus position accurately regardless of the absolute phase angles of their response profiles.

Only about half the simple cells in our sample were found to have Cartesian separable 2D spatial response profiles. Thus, for half the cells, application of conventional 1D length and width response measures would not yield an accurate 2D description of the receptive field. There are many ways in which $2 \mathrm{D}$ receptive fields could fail to be Cartesian separable (consider curved or nonparallel subregions), but we found a single phenomenological substrate for this effect. In each case, nonseparability arose from the staggered placement of individual subregions so that they were offset along an axis parallel to their long dimensions.

It is not obvious what significance this surprising receptive-field organization has in terms of neural image representation. Under the assumption of spatial linearity, it will have impact on the responses of these cells in the 2D spatial frequency domain. Through the use of the 2D Fourier transform, it can be shown that Cartesian separable 2D spatial response profiles of the type we have observed should yield elliptical 2D spectral response profiles, which are radially symmetric and Cartesian separable (subject to the appropriate rotation of coordinates). Similarly, 2D spatial response profiles with staggered subregions (not Cartesian separable) should yield 2D spectral response profiles, which are not Cartesian separable regardless of coordinate rotations (it will be elliptical but will not point toward the origin, Ref. 44). This issue will be addressed in the following paper (19).

Many of the new features of simple receptive-field structure that we have demonstrated in this paper can be captured in caricature. A receptive-field sketch as they are often published is shown in Fig. 13A. The other sketches (Fig. 13, $B-F$ ) reflect additional aspects of simple receptive fields as found in this study: I) subregions wax and wane smoothly in every direction (no abrupt edges, no corners); 2) the subregions within single receptive fields often vary in length but less so in width; 3) spatial phase is continuously variable (not just odd and even symmetry); and 4) simple receptive fields are found in both Cartesian separable and nonseparable forms.

These observations place obvious constraints on functional models of simple receptive fields. Any such model must be smooth and continuous everywhere, must permit noncanonical spatial phase, must be explicitly 2D, and must permit Cartesian separable as well as nonseparable forms.

\section{Reverse correlation}

Many investigators have used traditional stimulus-response methods to characterize various visual receptive-field properties, usually using one or more PST histograms $(11,43)$. This can be viewed as a form of forward correlation in which the probability of a spike is calculated as a function of time relative to stimulus onset and/or offset. By judicious choice of the stimulus ensemble, this approach has been used to estimate the 1D spatial impulse response of visual neurons $(7,9,20,27,29,30,33,34,37,43)$. However, this approach tends to be inefficient; our efforts to apply forward correlation 
A
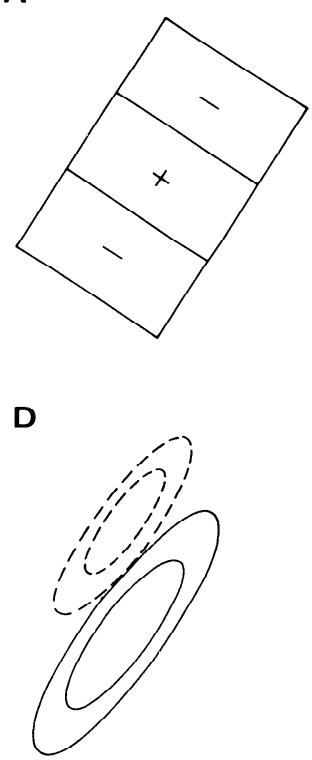

B

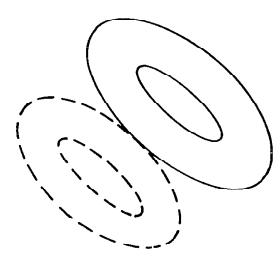

C

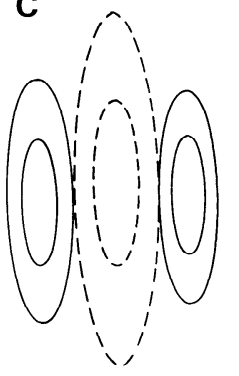

$\mathbf{F}$

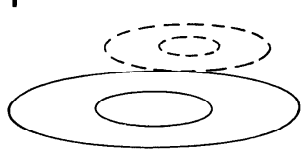

FIG. 13. Caricatures illustrate the observed variability in the two-dimensional spatial organization of simple receptive fields. $A$ : a simple receptive-field caricature as it is usually drawn suggests a Cartesian separable function in which the subregions are rectangular, have sharp boundaries, corners, are the same size, and of equal strength. $B$ : a simple receptive field in which the subregions are the same size, shape, and strength, but the corners have been rounded and 2 contours drawn to indicate that response amplitude varies smoothly in all directions. Subregions within a single receptive field may be of different lengths $(C, E, F)$, may be shifted with respect to the long axis of the field (not Cartesian separable, $D, F$ ), and may be of different strengths (noncanonical phase, $E, F$ ).

to $2 \mathrm{D}$ spatial characterization of simple receptive fields were hopelessly unsuccessful.

The less frequently used reverse correlation method finds the stimuli that precede spikes by given time intervals (the precvent stimulus ensemble of Eggermont et al., 8). Essentially, one obtains the same information: an estimate of the probability that a particular stimulus causes a spike. Again, by judicious choice of stimuli, the spatial impulse response can be obtained. Stimulus duration is not a limitation.

The method we have described may be thought of as a simplified subset on the even more powerful Gaussian white noise approach $(25,45)$. In a $2 \mathrm{D}$ reccptivc-ficld study using this method, all area elements would be present in each stimulus frame, with a Gaussian probability distribution in contrast. There are advantages and disadvantages to this approach. Its power lies in the ability to calculate second- and higher-order kernels, thus identifying and characterizing spatial and temporal nonlinearities within the receptive field. The problems are practi- cal. It is difficult to generate many simultaneous stimuli with good spatial and temporal resolution (with the Innisfree image generator it is impossible). There is considerable difficulty computing the cross-correlations due to the enormous image data base, which is itself a problem to maintain.

In our implementation of reverse correlation, only one stimulus is present at any given time. Using the Innisfree image generator, it is easy to present single stimuli with good spatial and temporal resolution. The first-order cross-correlation with this simplified stimulus ensemble, which is small enough to reside in the computer's memory, can be computed in real time. Other novel approaches designed to reduce computational overhead at the expense of generality have been used by several investigators. Notable among them are photographic reverse correlation using television snow as a stimulus and videotape as a mass storage medium (16), and the use of paired stimuli (28). We anticipate that paired stimuli distributed over two spatial dimensions would permit 
exploration of low-order multidimensional spatial nonlinearities and allow the extension of this method to complex cells.

The temporal resolution of this method could be improved. It is possible not only to record which stimulus was present at the time the spike occurred, but also the temporal delay between the beginning of the stimulus and the spike. This enhancement in the data structure allows calculation of the stimulus distribution preceding spikes at any fraction of the stimulus duration. We have implemented this improvement and have used it with a handful of cells. Examination of these reverse correlations with 1-ms temporal resolution is fully consistent with the spatial properties described in this study. The temporal properties are interesting in their own right and will be described elsewhere.

\section{The stimuli}

The stimuli used in these experiments to determine spatial response profiles depart from those generally used in visual physiology in that they were spatially noisy, delivered at high rates (usually $20 \mathrm{~Hz}$ ), and quite small. In addition, stationary stimuli, although used by several investigators $(27,33$, 34 ), have been discarded in favor of moving stimuli by others $(26,40)$.

In most investigations, stimuli are chosen that are guaranteed to produce high rates of discharge. Thus experiments are commonly designed with stimuli that take advantage of spatial summation within discharge zones and superposition of responses resulting from stimulus movement. We chose our stimuli for their analytic usefulness; they are small so that the fields could be explored in two spatial dimensions, presented briefly with no interstimulus interval so that useful

\section{REFERENCES}

1. Adelson, E. H. ANd Bergen, J. R. Spatio-temporal energy models for the perception of motion. $J$. Opt. Soc. Am. 2: 284-299, 1985.

2. ANDERSON, D. P. Hidden line elimination in projected grid surfaces. ACM Trans. Graphics 4: 274-288, 1982.

3. Bishop, P. O., CoOmbs, J. S., ANd Henry, G. H. Interaction effect of visual contours on the discharge frequency of simple striate neurons. J. Physiol. Lond. 219: 659-687, 1971.

4. Burr, D. C., Ross, J., And Morrone, M. C. Seeing objects in motion. Proc. R. Soc. Lond. B Biol. Sci. 227: 249-265, 1986.

5. DaUgman, J. G. Uncertainty relation for resolution responses could be acquired in reasonable times, and stationary so as not to confound spatial and temporal variables. As a consequence, we achieved very low firing rates and few spikes per stimulus, at best one or two. No correlation between the stimulus and the spike train could be detected by listening to the latter with an audio monitor.

Yet the results of this study demonstrate that very small changes in the probability of discharge can be measured even when the overall response rate is low. In fact, large responses may produce distortion due to saturation of the discharge mechanism and may mask an essentially simple (linear) system by driving it to (nonlinear) extremes. A preference for stimuli that elicit large responses is no more than a bias in experimental design.

Current terminology reflects this bias. A stimulus that under some narrow set of circumstances gives the largest response is said to be "optimal." But optimal implies "best", and although a large response is easier to observe, it is unlikely that it is "better" than a small one as far as the brain is concerned. To imagine that responses to optimal and nonoptimal stimuli are qualitatively different is to ignore an entire dimension of visual processing along with the richness of receptivefield structure that subserves it.

\section{ACKNOWLEDGMENTS}

We are grateful to A. Gottschalk and A. Aertsen for their comments on and criticisms of the method.

This work was supported by National Institutes of Health Grant NEI-R01-EY-04638.

Present address of J. P. Jones: Division of Engineering Physics and Mathematics, Oak Ridge National Laboratory, Oak Ridge, TN 37830.

Received 19 May 1986; accepted in final form 7 July 1987.

in space, spatial frequency, and orientation optimized by two-dimensional visual cortical filters. $J$. Opt. Soc. Am. 2: 1160-1169, 1985.

6. DEBOER, E. Reverse correlation. I. A heuristic introduction to the technique of triggered correlation with application to the analysis of compound systems. Proc. K. Ned. Akad. Wet. Ser. C Biol. Med. Sci. 71: 472-486.

7. DeValois, R. L., Albrecht, D. G., And ThorELL, L. G. Cortical cells: bar and edge detectors, or spatial frequency filters. In: Frontiers of Visual Science, edited by J. Cool and E. Smith. New York: Springer, 1978.

8. Eggermont, J. J., Johannesma, P. I. M., AND 
AERTSEN, A. M. H. J. Reverse-correlation methods in auditory research. Q. Rev. Biophys. 16: 341-414, 1983.

9. Emerson, R. C. And Gerstein, G. L. Simple striate neurons in the cat. I. Comparison of responses to moving and stationary stimuli. J. Neurophysiol. 40: 119-135, 1977.

10. FiElD, D. J. AND TolHuRst, D. J. The structure and symmetry of simple-cell receptive-field profiles in the cat's visual cortex. Proc. R. Soc. Lond. B Biol. Sci. 228: 379-400, 1986.

11. Gerstein, G. L. AND Kiang, N. Y. S. An approach to the quantitative analysis of electrophysiological data from single neurons. Biophys. J. 1: 15-28, 1960.

12. Glezer, V. D., Tsherbach, T. A., Gauselman, V. E., AND BONDARKO, V. M. Spatio-temporal organization of receptive-fields of the cat striate cortex. Biol. Cybern. 43: 39-45, 1982.

13. HeGGelund, P. Receptive field organization of simple cells in cat striate cortex. Exp. Brain Res. 42: 89-98, 1981.

14. HENRY, G. H. Receptive field classes of cell in the striate cortex of the cat. Brain Res. 133: 1-28, 1977.

15. Henry, G. H., GoOdwin, A. W., AND Bishop, P. O. Spatial summation of responses in receptive fields of single cells in cat striate cortex. Exp. Brain Res. 32: 245-266, 1978.

16. Hida, E., NAKa, K.-I., AND YOKOYAMA, K. A new method for mapping spatio-temporal receptive field using television snow stimulation. $J$. Neurosci. Methods 8: 225-230, 1983.

17. Hubel, D. H. AND WiESEL, T. N. Receptive fields, binocular interaction and functional architecture in the cat's visual cortex. J. Physiol. Lond. 160 106-154, 1962

18. JONES, J. P. AND PALMER, L. A. An evaluation of the two-dimensional Gabor filter model of simple receptive fields in cat striate cortex. $J$. Neurophysiol. 58: 1233-1258, 1987.

19. JONeS, J. P., STePnoski, A., AND PAlmer, L. A. The two-dimensional spectral structure of simple receptive fields in cat striate cortex. $J$. Neurophysiol. 58: 1212-1232, 1987.

20. KULIKOWSKI, J. J. AND BISHOP, P. O. Linear analysis of the response of simple cells in the cat visual cortex. Exp. Brain Res. 44: 386-400, 1981.

21. Kulikowski, J. J., BISHOP, P. O., AND KaTO, H. Spatial arrangement of responses by cells in the cat visual cortex to light and dark bars and edges. Exp. Brain Res. 44: 371-385, 1981.

22. Kulikowski, J. J., Marcelja, S., AND Bishop, P. O. Theory of spatial position and spatial frequency relations in the receptive fields of simple cells in the cat's striate cortex. Biol. Cybern. 43: 187-198, 1982.

23. LEVICK, W. R. Another tungsten microelectrode. Med. Biol. Eng. 10: 510, 1972.

24. MarcelJA, S. Mathematical description of the responses of simple cortical cells. J. Opt. Soc. Am. 70 1297-1300, 1980.

25. Marmarelis, P. Z. AND Marmarelis, V. Z. Analysis of Physiological Systems. New York: Plenum, 1978.

26. Maske, R., Yamane, S., And Bishop, P. O. Simple and B-cells in cat striate cortex. Complementarity of responses to moving light and dark bars. J. Neurophysiol. 53: 670-685, 1985.

27. Movshon, J. A., Thompson, I. D., And TolHURST, D. J. Spatial summation in the receptive fields of simple cells in the cat's striate cortex. $J$. Physiol. Lond. 283: 53-77, 1978.

28. Movshon, J. A., Thompson, I. D., AND TOLHURST, D. J. Receptive field organization of complex cells in the cat's striate cortex. J. Physiol. Lond. 283: 79-99, 1978.

29. Mullikin, W. H., Jones, J. P., AND Palmer, L. A. Receptive-field properties and laminar distribution of X-like and Y-like simple cells in cat area 17. $J$. Neurophysiol. 52: 350-371, 1984.

30. Mullikin, W. H., Jones, J. P., AND Palmer, L. A. Periodic simple cells in cat area 17. J. Neurophysiol. 52: 372-387, 1984.

31. Orban, G. A. Neuronal Operations in the Visual Cortex. Berlin: Springer-Verlag, 1984.

32. Orban, G. A., Kato, H., AND Bishor, P. O. Endzone region in receptive fields of hypercomplex and other striate neurons in the cat. J. Neurophysiol. 42: 818-832, 1979.

33. ORBAN, G. A. AND KENNEDY, H. The influence of eccentricity on receptive field types and orientation selectivity in area 17 and 18 of the cat. Brain Res. 208: 203-208, 1981

34. Palmer, L. A. AND Davis, T. L. Receptive-field structure in cat striate cortex. J. Neurophysiol. 46: 260-276, 1981

35. Palmer, L. A., Jones, J. P., AND GotTschalk, A. Constraints on the estimation of spatial receptive field profiles of simple cells in visual cortex. In: $A d$ vanced Methods of Physiological System Modelling, edited by V. Marmarelis. Los Angeles, CA: Biomedical Simulations Resource, 1987.

36. POllen, D. A. AND RoNNER, S. F. Phase relationships between adjacent simple cells in the visual cortex. Science Wash. DC 212: 1409-1411, 1981.

37. Pollen, D. A. AND RonNer, S. F. Spatial computation performed by simple and complex cells in the visual cortex of the cat. Vision Res. 22: 101-118.

38. Pollen, D. A. AND Ronner, S. F. Visual cortical neurons as localized spatial frequency filters. IEEE Trans. Syst. Man Cyber. SMC-13: 907-916, 1983.

39. Rybicki, G. B., TraCy, D. M., AND Pollen, D. A. Complex cell response depends on interslit spacing. Nat. New Biol. 240: 77-78, 1972.

40. SChiller, P. H., Finlay, B. L., AND Volman, S. F. Quantitative studies of single-cell properties in monkey striate cortex. I. Spatiotemporal organization of receptive fields. J. Neurophysiol. 39: 1288-1319, 1976.

41. SChumer, R. A. And Movshon, J. A. Length summation in simple cells of cat striate cortex. $\mathrm{Vi}$ sion Res. 24: 565-571, 1984.

42. SNYDER, W. V. Algorithm 531. Contour plotting. ACM Trans. Math. Software 4: 290-294, 1978.

43. Stevens, J. K. AND Gerstein, G. L. Spatiotemporal organization of cat lateral geniculate receptive fields. J. Neurophysiol. 39: 213-238, 1976.

44. SZULbORSKI, R., STEPNOSKI, R., JONES, J. P., AND PALMER, L. A. Separability assumptions in simple receptive fields. Soc. Neurosci. Abstr. 11: 651, 1985.

45. Wiener, N. Nonlinear Problems in Random Theory. New York: Wiley, 1958. 